\title{
Epigenetic signals that direct cell type specific interferon beta response in mouse cells
}

Markus Muckenhuber ${ }^{1,2}$, Isabelle Lander ${ }^{1,2}$, Katharina Müller-Ott ${ }^{1,3}$, Jan-Philipp Mallm ${ }^{1,4}$, Lara C. Klett $^{1,2}$, Caroline Knotz ${ }^{1}$, Jana Hechler ${ }^{1,5}$, Nick Kepper ${ }^{1,6}$, Fabian Erdel ${ }^{1,7}$, Karsten Rippe ${ }^{1,{ }^{*}}$

${ }^{1}$ Division of Chromatin Networks, German Cancer Research Center (DKFZ) and Bioquant, Heidelberg, Germany

${ }^{2}$ Faculty of Biosciences, Heidelberg University, Germany

${ }^{3}$ Present address: Illumina Centre, Granta Park, Cambridge, UK

${ }^{4}$ Single Cell Open Lab, German Cancer Research Center (DKFZ), Heidelberg, Germany

${ }^{5}$ Present address: Technische Universität Nürnberg, Nürnberg, Germany

${ }^{6}$ Present address: Bioquant, Heidelberg University, Heidelberg, Germany

${ }^{7}$ Present address: MCD, Centre de Biologie Intégrative (CBI), University of Toulouse, CNRS, Toulouse, France

* Correspondence: Karsten Rippe, e-mail: karsten.rippe@dkfz.de

Running title: Chromatin context dependent interferon response

Key words: STAT transcription factor, gene regulation, histone modifications, innate immune response, RNA-seq, ChIP-seq, scATAC-seq 


\section{Abstract}

The antiviral response induced by type I interferon (IFN) via the JAK-STAT signaling cascade activates hundreds of IFN-stimulated genes (ISGs). While this response occurs essentially in all human and mouse tissues it varies between different cell types. However, the linkage between the underlying epigenetic features and the ISG pattern of a given cell is not well understood. We mapped ISGs, binding sites of the STAT1 and STAT2 transcription factors and chromatin features in three different mouse cell types (embryonic stem cells, neural progenitor cells and embryonic fibroblasts) before and after treatment with IFN $\beta$. The analysis included gene expression, chromatin accessibility and histone $\mathrm{H} 3$ lysine modification by acetylation (ac) and mono-/tri-methylation (me1, me3). A large fraction of ISGs and STAT binding sites were cell type specific with promoter binding of a STAT1STAT2 complex (STAT1/2) being a key driver of ISG induction. Furthermore, STAT1/2 binding to putative enhancers at intergenic and intronic sites induced ISG expression as inferred from a chromatin co-accessibility analysis. STAT1/2 binding was dependent on the chromatin context and positively correlated with pre-existing H3K4me1 and H3K27ac marks in an open chromatin state while the presence of $\mathrm{H} 3 \mathrm{~K} 27 \mathrm{me} 3$ had an inhibitory effect. Thus, chromatin features present before stimulation represent an additional regulatory layer for the cell type specific antiviral response.

\section{Introduction}

Type I interferons (IFNs) like IFN $\alpha$ and IFN $\beta$ are expressed across almost all tissues in human and mouse as a first line of defense against viral infections (Hoffmann et al, 2015; Lazear et al, 2019; Sa Ribero et al, 2020; Stanifer et al, 2020). They activate hundreds of IFN-stimulated genes (ISGs) during innate immune response. Virus infection induces IFN $\beta$ in most cell types, which then can stimulate production of other type I IFNs (Hoffmann et al, 2015). The ISG activation by IFN is not uniform but occurs in a cell type specific manner (Lazear et al, 2019; Sa Ribero et al, 2020; Stanifer et al, 2020) and displays striking changes during differentiation of human embryonic stem cells (Wu et al, 2018). Mouse embryonic stem cells (ESCs) do not express IFN themselves upon viral infection but respond to IFN and display an attenuated innate immune response as compared to differentiated murine cells (D'Angelo et al, 2016; Gonzalez-Navajas et al, 2012; Guo, 2017; Guo et al, 2015; Wang et al, 2014; Wang et al, 2013; Whyatt et al, 1993).

One aspect of the cell type specific response to IFNs are specific epigenetic features that modulate ISG activation via the JAK-STAT signaling cascade. This pathway involves phosphorylation of STAT1 and STAT2 transcription factors that, together with IRF9, assemble into the IFN-stimulated gene factor 3 (ISGF3) complex (Chen et al, 2017; Hu et al, 2021; Ivashkiv \& Donlin, 2014; Stark \& Darnell, 2012; Villarino et al, 2017). ISGF3 translocates into the nucleus, binds interferon-stimulated response elements (ISREs) and activates ISGs. In addition, IFNy activation sites (GAS) are bound predominantly by phosphorylated STAT1 homodimers and can drive IFN mediated gene induction. The STAT binding sites are frequently located at promoters and regulatory sites such as enhancers (Begitt et al, 2014; Ostuni et al, 2013; Vahedi et al, 2012). Chromatin remodeling complexes, histone acetyltransferases and deacetylases can act as modulators for the downstream JAK-STAT signaling cascade (Au-Yeung \& Horvath, 2018; Chen et al, 2017; Liu et al, 2002; Nusinzon \& Horvath, 2003; Testoni et al, 2011; Villarino et al, 2017). However, it is not well understood how specific chromatin features affect STAT1 and STAT2 binding and ISG induction. Here, we dissected the cell type 
specific IFN $\beta$ response by comparing mouse ESCs, neural progenitor cells (NPCs) derived by in vitro ESC differentiation and mouse embryonic fibroblasts (MEFs) in a comprehensive genome-wide analysis. Our sequencing-based readouts comprised transcription, binding of STAT1 and STAT2, acetylation (ac) and mono- and tri-methylation (me1, me3) of histone $\mathrm{H} 3$ lysine residues (H3K4me1, H3K4me3, H3K9ac, H3K27ac, H3K9me3 and H3K27me3) and open chromatin mapped by the assay for transposase-accessible chromatin (ATAC). The resulting sets of common and cell type specific ISGs were linked to the binding of a STAT1-STAT2 complex (STAT1/2) at promoters and enhancers in dependence of their chromatin state. Our analysis sheds light on the interplay of epigenetic signals, STAT1/2 binding at cis-regulatory elements and the cell type specific modulation of innate immune response.

\section{Results}

\section{IFN $\beta$ induces anti-viral gene expression programs in all three cell types}

ESCs, MEFs and NPCs were obtained from a 129/Ola mouse strain and represent an established cellular system that allowed us to compare the cell type dependent epigenetic makeup and IFN $\beta$ response of the same genome for a large number of chromatin features (Molitor et al, 2017; Teif et al, 2012) (Fig. 1A). The three different cell types were treated with IFN $\beta$ for $1 \mathrm{~h}$ or $6 \mathrm{~h}$ and gene expression profiles were acquired by RNA sequencing (RNA-seq) (Supplementary Table S1). Differential gene expression analysis identified in total 191 ISGs induced in ESCs, 463 ISGs in MEFs, and 244 ISGs in NPCs over unstimulated controls (0 h) (Fig. 1B, Supplementary Table S2, Supplementary Data Set 1). As expected, a GO-term analysis yielded upregulated genes related to anti-viral programs and innate immune responses in all three cell types (Supplementary Fig. 1A). By intersecting the three individual ISG sets, we obtained 143 common ISGs while 33 (ESC), 17 (NPC) and 221 (MEF) ISGs were cell type specific (Fig. 1C, Supplementary Data Set 1). The ISGs found in NPCs mainly represented a subset of MEF ISGs (227 of 244) pointing to a high similarity of the IFN $\beta$ response in NPCs and MEFs (Fig. 1C). A differential gene expression analysis of only intronic reads to assess nascent RNA levels gave very similar results with a somewhat lower number of ISGs detected in ESCs (Supplementary Fig. 1B, C; Supplementary Table S2). We conclude that changes induced by IFN $\beta$ occurred predominantly at the gene expression level with only minor differences in RNA stability.

\section{IFN $\beta$ response is mostly homogenous at the single-cell level}

We assessed by single-cell RNA sequencing (scRNA-seq) if the transcriptional response in ESCs and MEFs was homogeneous or if the observed upregulation of ISGs arises from a subset of strongly responding cells (Fig. 1D; Supplementary Fig. 1D). In contrast to ESCs, MEFs consistently formed two distinct clusters (clusters 0 and 1 and clusters 2 and 3, respectively) in the single-cell embedding of transcriptomic profiles. This clustering arose from upregulated genes associated with KEGG pathway "extra cellular matrix receptor interaction" in clusters 0 and 2 as opposed to the "focal adhesion" KEGG pathway in clusters 1 and 3 (Supplementary Fig. 1E, F). Based on these expression profiles we annotated clusters 0 and 2 as "mesenchymal-like" and clusters 1 and 3 as "epithelial-like". 

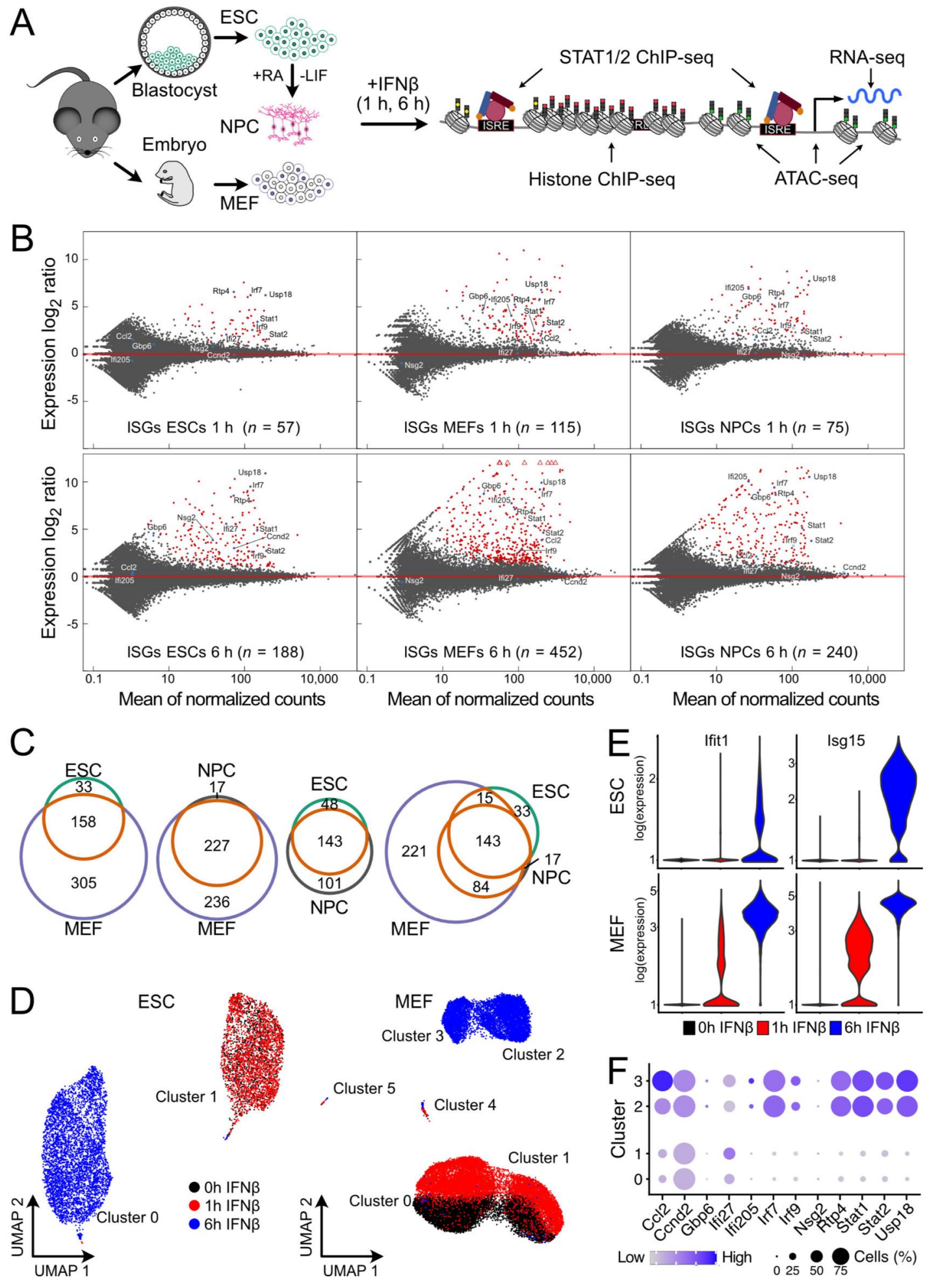

Figure 1. ISG induction patterns in ESCs, MEFs and NPCs. (A) ESCs, NPCs differentiated in vitro from them and MEFs from a 129/Ola mouse strain were studied to reveal the relation between cell type specific chromatin features and IFN $\beta$ response. (B) Gene expression changes after IFN $\beta$ treatment. Red dots represent significant differentially expressed genes at $p_{\text {adj }}<0.05$ and fold change $\geq 1.5$. Four biological replicates for ESCs, two for MEFs, and four for NPCs were acquired for RNAseq. (C) Overlap of ISGs found after $1 \mathrm{~h}$ or $6 \mathrm{~h}$ IFN $\beta$ treatment in ESCs, NPCs and MEFs. (D) Singlecell embedding of gene expression in ESCs (left) and MEFs (right). (E) Normalized expression levels of the ISGs Ifit1 and Isg15 in single ESCs (top) and MEFs (bottom). Both genes were reliably detected as ISGs in the bulk RNA-seq analysis after $1 \mathrm{~h}$. (F) Expression levels of selected ISGs identified by bulk RNA-seq data according to aggregated scRNA-seq in MEF clusters 0, 1, 2 and 3. 

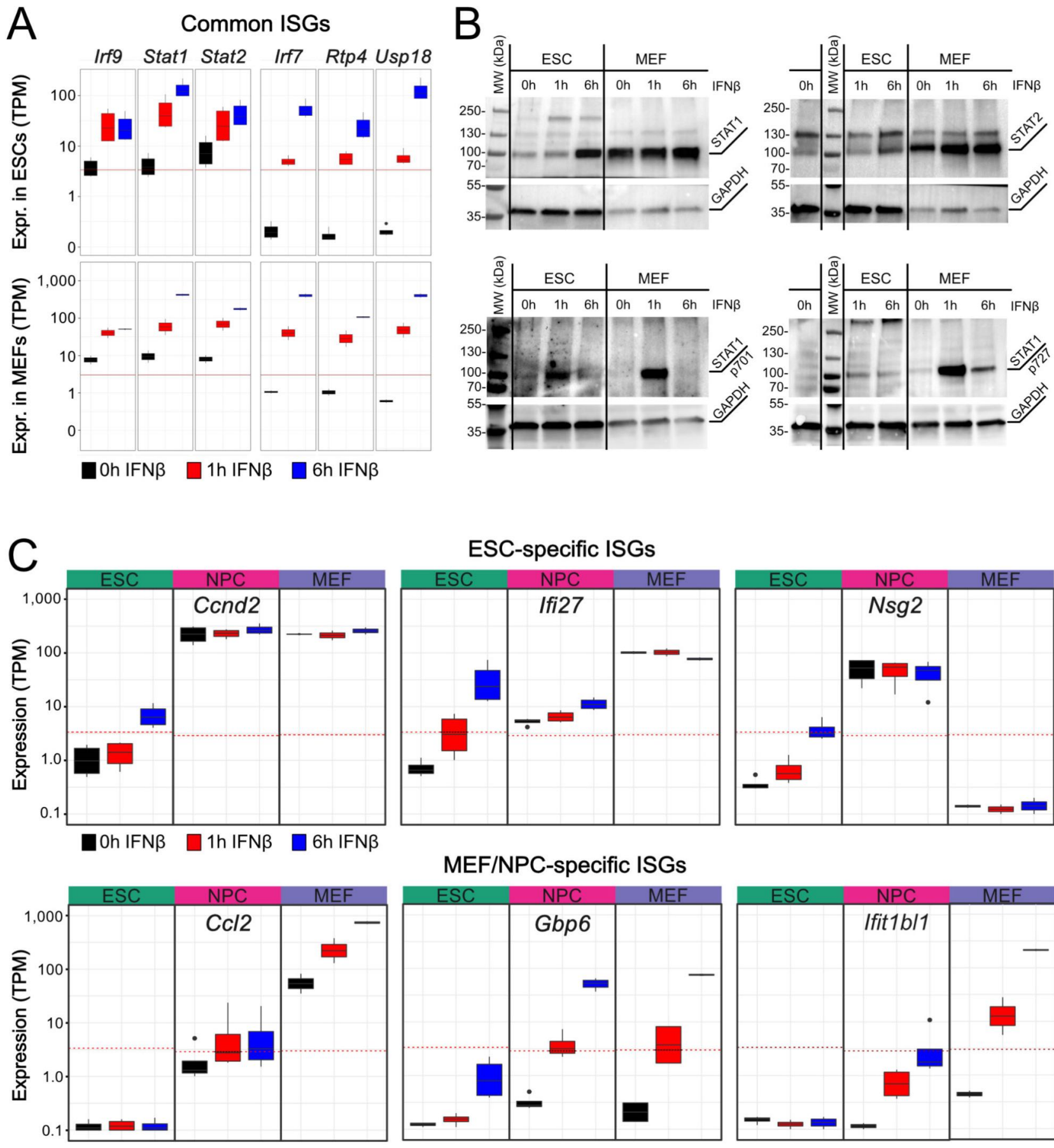

MEF/NPC-specific ISGs

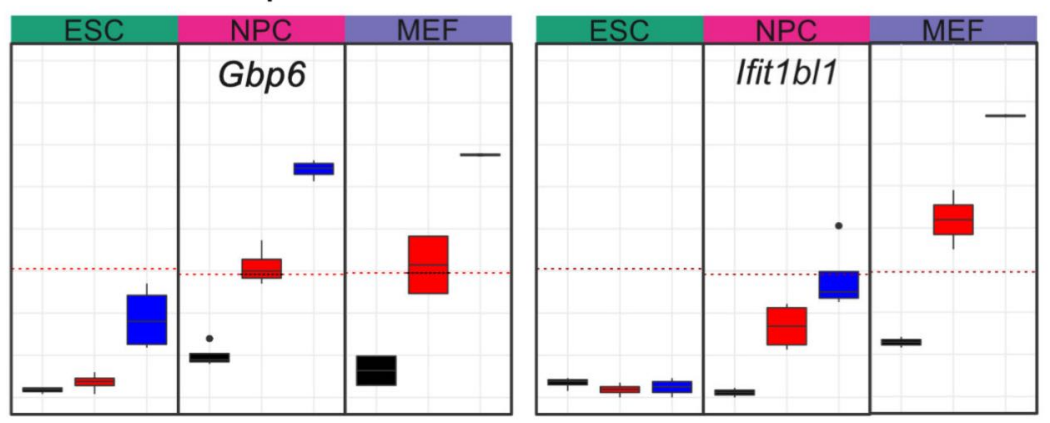

Figure 2. Cell type specific ISG induction and protein expression. (A) Normalized gene expression levels of selected ISGs from bulk RNA-seq in ESCs (top) and MEFs (bottom). Gene expression is given as transcripts per kilobase million (TPM). (B) Western blots of IFN $\beta$ stimulated ESCs and MEFs at $0 \mathrm{~h}, 1 \mathrm{~h}$ and $6 \mathrm{~h}$ time points. The top row shows total levels of STAT1 (left) and STAT2 (right). The lower row shows phosphorylation of STAT1 at position 701 (left) and 727 (right). GAPDH was used as housekeeper gene control. (C) Normalized gene expression levels of selected cell type-specific ISGs in ESCs, NPCs and MEFs. The red line represents a cell type-specific threshold to distinguish active and repressed genes. Top: Expression of ISGs Ccnd2, Ifi27 and Nsg2 was only induced in ESCs. Bottom: Expression of ISG Ccl2 was induced in MEFs. Expression of ISGs Gbp6 and Ifit1b/1 was induced in MEFs and NPCs. 
Inspection of the UMAP plots showed no separate clustering of untreated $(0 \mathrm{~h})$ and $1 \mathrm{~h}$ IFN $\beta$ treated ESCs, while cells after $6 \mathrm{~h}$ stimulation formed a distinct cluster. Untreated and $1 \mathrm{~h}$ IFN $\beta$ treated MEFs separated within the same clusters while MEFs treated for $6 \mathrm{~h}$ with IFN $\beta$ were present in a separate cluster again. The pattern was in line with the relatively small global transcriptomic changes in ESCs and MEFs after $1 \mathrm{~h}$ IFN $\beta$ treatment, where 57 and 115 ISGs were detected by bulk RNAseq as compared to 188 and 452 genes after $6 \mathrm{~h}$ for ESCs and MEFs, respectively (Fig. 1B). The scRNA-seq response pattern was illustrated for two ISGs, Ifit1 and Isg15 (Fig. 1E). The number of cells where transcripts of the two genes were detected, largely increased from the $1 \mathrm{~h}$ to the $6 \mathrm{~h}$ time point as more RNA is produced. We conclude that the apparent heterogeneity after $1 \mathrm{~h}$ IFN $\beta$ appears to arise to a significant extend from the reduced detection sensitivity of scRNA-seq for lowly expressed genes that show an increased drop-out frequency (Yamawaki et al, 2021). Furthermore, the expression patterns and IFN $\beta$ response dynamics of the two MEF clusters (cluster 0 vs 1; cluster 2 vs 3) were highly similar in terms of ISGs and their induced gene expression levels (Fig. 1F). Thus, the IFN $\beta$ response was rather homogeneous in the two different cell types at the single-cell level and we used the ISG definition from the bulk RNA-seq analysis for further analysis.

\section{ISG expression varies between cell types in response strength and specificity}

Next, we compared the transcriptional response to IFN $\beta$ in the three cell types in further detail. The distribution of gene expression levels in non-stimulated cells was fitted with distributions for active and repressed genes to define a background threshold for evaluation of differences in the IFN $\beta$ response (Supplementary Fig. 2A). In ESCs and MEFs some genes like Irf9, Stat1 and Stat2 were already lowly expressed in unstimulated cells and showed a significant increase in expression after IFN $\beta$ treatment (Fig. 2A). Other ISGs like Irf7, Rtp4 and Usp18 changed from a repressed to an activated state after IFN $\beta$ stimulation. Compared to ESCs, MEFs displayed a 10 to 100 -fold stronger induction of these common ISGs, which is in line with previous findings (Wang et al, 2014). To further dissect the overall stronger response in MEFs, we compared expression levels of factors of the IFN signaling pathway. The Ifnar1 and Ifnar2 receptors as well as Jak1 kinase were higher expressed in MEFs than in ESCs while for key transcription factors Stat1, Stat2 and Irf9 no differences were identified (Supplementary Fig. 2B). A western blot with STAT1 and STAT2 antibodies showed that STAT1 and STAT2 proteins were present at lower levels in ESCs before and after IFN $\beta$ induction as compared to MEFs (Fig. 2B). The amount of STAT1 phosphorylated at residue $701^{\text {(STAT1 }}$ p01) or 727 (STAT1 1 721) was clearly increased after $1 \mathrm{~h}$ in MEFs as compared to ESCs and decayed to low levels at the $6 \mathrm{~h}$ time point. Thus, we conclude that the globally attenuated response to IFN $\beta$ in ESCs involved epigenetic networks that lead to a reduced expression of key components of the JAK/STAT signaling pathway as compared to differentiated cells. The lower levels of active STAT1/2 protein complexes upon IFN $\beta$ induction are apparent from the comparing the amounts of STAT $1_{\text {p701 }}$ and STAT1 $1_{\text {p727 }}$ between ESCs and MEFs. In addition to these global differences, cell type specific differences were apparent as illustrated for selected genes in Fig. 2C. After 6 h of stimulation Ccnd2, Ifi27, and Nsg2 were induced in ESCs. In NPCs, Ccnd2 and Nsg2 were constitutively expressed while the lowly expressed Ifi27 showed only a small expression increase after $6 \mathrm{~h}$. In MEFs, expression of all three genes was not upregulated. In contrast, Cc/2, Gbp6 and Ifit1b/1 were specifically upregulated in MEFs upon IFN $\beta$ stimulation. Gpb6 and Ifit1b/1 also showed a response in NPCs albeit at a lower level. Gbp6 was lowly induced in ESCs but only after $6 \mathrm{~h}$. In summary, 
large cell type specific differences in gene expression levels were observed upon IFN $\beta$ stimulation between the three cell types that involved the expression of distinct sets of ISGs in ESCs and MEFs with NPCs showing a pattern that was similar to MEFs.

\section{STAT1/2 binding is cell type specific and correlates with ISG activation}

The differences in IFN $\beta$ response raise the question why certain ISGs were preferably expressed in one cell type and not in the other. To reveal molecular details of gene expression regulation we mapped STAT1 101 and STAT2 binding by chromatin immunoprecipitation followed by sequencing (ChIP-seq). Antibodies against STAT1 $1_{\mathrm{p} 701}$ and STAT2 in ESCs and MEFs were used with exemplary regions enriched for both transcription factors shown in Fig. 3A and the number of peaks detected given in Supplementary Table S2. A total of 208 peaks in ESCs and 276 peaks in MEFs were bound simultaneously by both transcription factors (Fig. 3B, Supplementary Table S2, Supplementary Data Set 2). These loci were annotated as "STAT1/2" binding sites in our analysis. They were likely to represent the ISGF3 complex as it has been shown previously, that STAT1 and STAT2 assemble with IRF9 to form the ISGF3 complex upon IFN stimulation (Platanitis et al, 2019). A total of 392 STAT1/2 binding sites were determined from the combined data set of ESCs and MEFs after $1 \mathrm{~h}$ and $6 \mathrm{~h}$ of IFN $\beta$ stimulation. The remaining peaks that only had STAT $1_{\mathrm{p} 701}$ or STAT2 bound were classified as "STAT1" and "STAT2" binding sites, respectively. The overlap of peaks between cell types was moderate (Fig. 3E). Only 38 sites were found to be bound by STAT1 in both cell types, while most STAT2 peaks were cell type specific. STAT1/2 binding sites common to both cell types comprised $44 \%$ (ESC) and 33\% (MEFs) of the peaks. To validate the peak specificity, we determined enriched known motifs in STAT binding sites. In both ESCs and MEFs, the STAT-family motifs (STAT1, STAT3, STAT3 + IL21, STAT4, STAT5) were enriched at STAT1 peaks, while IRFfamily motifs (IRF1, IRF2, IRF3, IRF8, ISRE) were the most enriched motifs in STAT1/2 and STAT2 peaks (Fig. 3C). Due to the high similarity of motifs within each family, we defined all detected STAT peaks with at least one of these to be a specific peak (Supplementary Fig. 3A). At least one of these family motifs was found in $66 \%$ (STAT1), $83 \%$ (STAT1/2) and $86 \%$ (STAT2) of the ESC peaks and $85 \%$ (STAT1), $90 \%$ (STAT1/2) and $88 \%$ (STAT2) of the MEF peaks. Thus, the same motifs were recognized independent of cell type and in line with the classification into STAT1, STAT2 and STAT1/2 binding sites. This conclusion was corroborated by a de novo motif analysis (Supplementary Fig. 3B-C). The top de novo motif was in all groups one of the STAT or IRF family with a similarity score of $\sim 0.9$. It is noted that the total number of the 1,885 STAT peaks detected by ChIP-seq represents only a minor fraction of the approximately 2.5 million STAT- or IRF-family sequence motifs in the mouse genome ( $\sim .8$ million IRF motifs, 1.7 million STAT motifs). Based on these findings, we conclude that the DNA sequence is neither sufficient to predict the experimentally observed STAT binding sites nor can it rationalize the differences in STAT binding sites detected between cell types. 

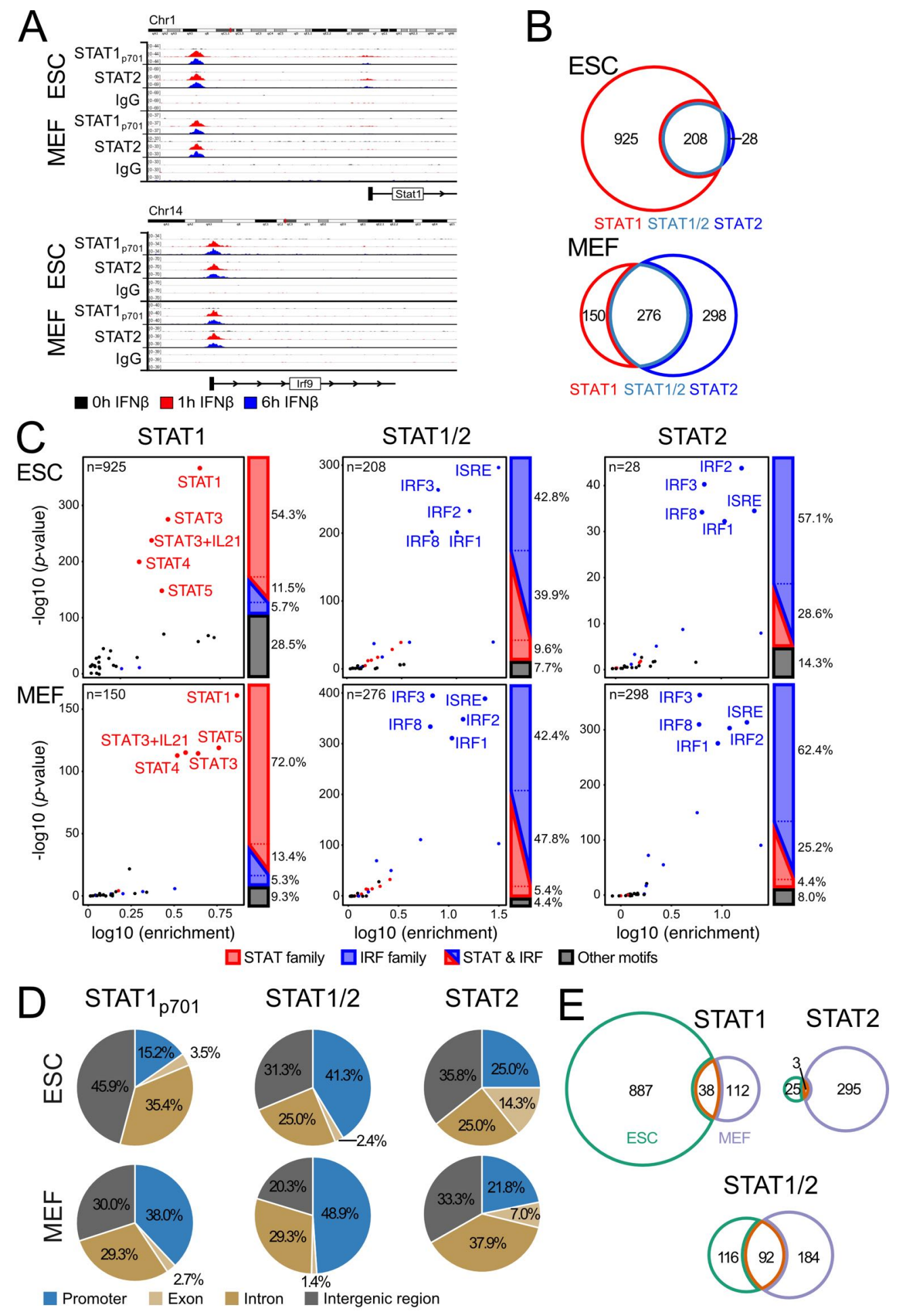

Figure 3. Cell type specific binding of STAT1 and STAT2. (A) ChIP-seq of STAT1 1 p701 and STAT2 in the genomic regions upstream of Stat1 (left) and at the promoter of Irf9 (right). Tracks show one representative replicate for each condition. (B) STAT1 $1_{\mathrm{p} 701}$ and STAT2 peaks in ESCs and MEFs. The overlap of STAT1 $1_{\text {p701 }}$ and STAT2 peaks defined STAT1/2 binding sites. (C) Enrichment of transcription factor binding motifs in STAT1 $1_{\text {p701 }}$, STAT1/2 and STAT2 peak sets identified in ESCs and MEFs. Motif color scheme: STAT-family (STAT1, STAT3, STAT3+IL21, STAT4, STAT5), red; IRF-family (IRF1, IRF2, IRF3, IRF8 and ISRE (IRF9)), blue; other, black. Four biological replicates for ESCs and two for MEFs were analyzed. (D) Distribution of STAT $1_{\mathrm{p} 701}$, STAT1/2 and STAT2 peaks at promoters, exons, introns and intergenic regions annotated from the ENSEMBL data base. (E) Overlap of STAT binding sites between ESCs and MEFs for STAT1 ${ }_{\mathrm{p} 701}, \mathrm{STAT} 1 / 2$ and STAT2. 


\section{ISG activation can be partly assigned to STAT promoter binding}

To gain further insight in the activation mechanism of ISGs, we analyzed the spatial relation between STAT binding sites and ISGs. Almost half of the STAT1/2 peaks in ESCs and MEFs were located at promoters (defined as a window of $\pm 1 \mathrm{~kb}$ around the transcription start site) with around $3 / 4$ of them at ISGs (Fig. 3D, Supplementary Fig. 3D, Supplementary Data Set 3). In contrast, a smaller fraction of $15-38 \%$ of the STAT1 or STAT2 only peaks were at promoters. In addition, the promoters that displayed STAT1 binding but lacked STAT2 were mostly highly expressed genes and only a minor fraction of $6 \%$ in ESCs and $16 \%$ in MEFs were at ISGs. This fraction was around $50 \%$ for the STAT2 only peaks. Based on this analysis we conclude that STAT1/2 binding (representing bona fide ISGF3 complexes together with IRF9) at promoters was the main driver of ISG activation in our system ( $n=71$ in ESCs; $n=112$ in MEFs). In addition, ISG activation was provided for a smaller fraction of promoters by STAT2 in the absence of STAT1, in line with the conclusion that the STAT2IRF9 complex alone could provide some activation (Platanitis et al, 2019) ( $n=5$ in ESCs; $n=34$ in MEFs). STAT1 without STAT2 appeared to lack significant activation capacity in our system but rather displayed some propensity to bind to already active promoters. Nevertheless, it could potentially be involved in promoting transcription of some ISGs where it was found at the promoter ( $n=10$ in ESCs; $n=11$ in MEFs). For a remaining fraction of 105 (ESCs) and 306 (MEFs) ISGs, no STAT binding at the promoter was detected. Accordingly, these ISGs were either secondary target genes or become activated from non-promoter STAT binding sites. Based on these findings, we focused on STAT1/2 binding sites as a proxy for the ISGF3 complex to further characterize the relation between non-promoter STAT1/2 binding and ISGs.

\section{STAT1/2 enhancers are predicted from co-accessibility analysis}

The non-promoter STAT1/2 peaks at intronic or intergenic sites could represent enhancer elements that regulate ISGs from a distance. A simple assignment of these potential enhancer sites to the nearest gene linked these sites to only a few additional ISGs that lacked promoter bound STAT1/2 ( $n=13$ in ESCs; $n=41$ in MEFs) (Supplementary Fig. 4A). Thus, the assumption that the majority of enhancer targets can be predicted by selecting the closest gene appeared not be justified in our system. To further characterize potential targets of STAT1/2 binding at putative enhancers, we applied a novel strategy to define co-regulatory sites using co-accessibility events from single-cell ATAC (scATAC-seq) data (Fig. 4A-C, Supplementary Fig. 4B, Supplementary Table S3). The single-cell embeddings of chromatin accessibility showed no clear separation of ESCs and MEFs after IFN $\beta$ treatment (Fig. 4A), indicating that the observed gain in chromatin accessibility at STAT1/2 sites was not accompanied by a global alteration of the chromatin landscape (Supplementary Fig. 4B). These observations were in agreement with the RNA-seq data, where a couple of hundred ISGs were identified. For MEFs, two separate cell clusters were detected (Fig. 4B) and assigned to epithelial- and mesenchymal-like MEF subtypes by integration with the scRNAseq data (Fig. 1D, Fig. 4C). Next, we computed correlations between pairs of genomic loci that were simultaneously accessible in the same cell based on previously described approaches (Granja et al, 2021; Mallm et al, 2019) to detect enhancers with STAT1/2 linked to ISGs before and after IFN $\beta$ stimulation (Supplementary Fig. 4C, Supplementary Data Set 3). This analysis was conducted for 392 STAT1/2 binding sites in ESCs and MEFs with all $2 \mathrm{~kb}$ peaks in a surrounding $1 \mathrm{Mb}$ region. 

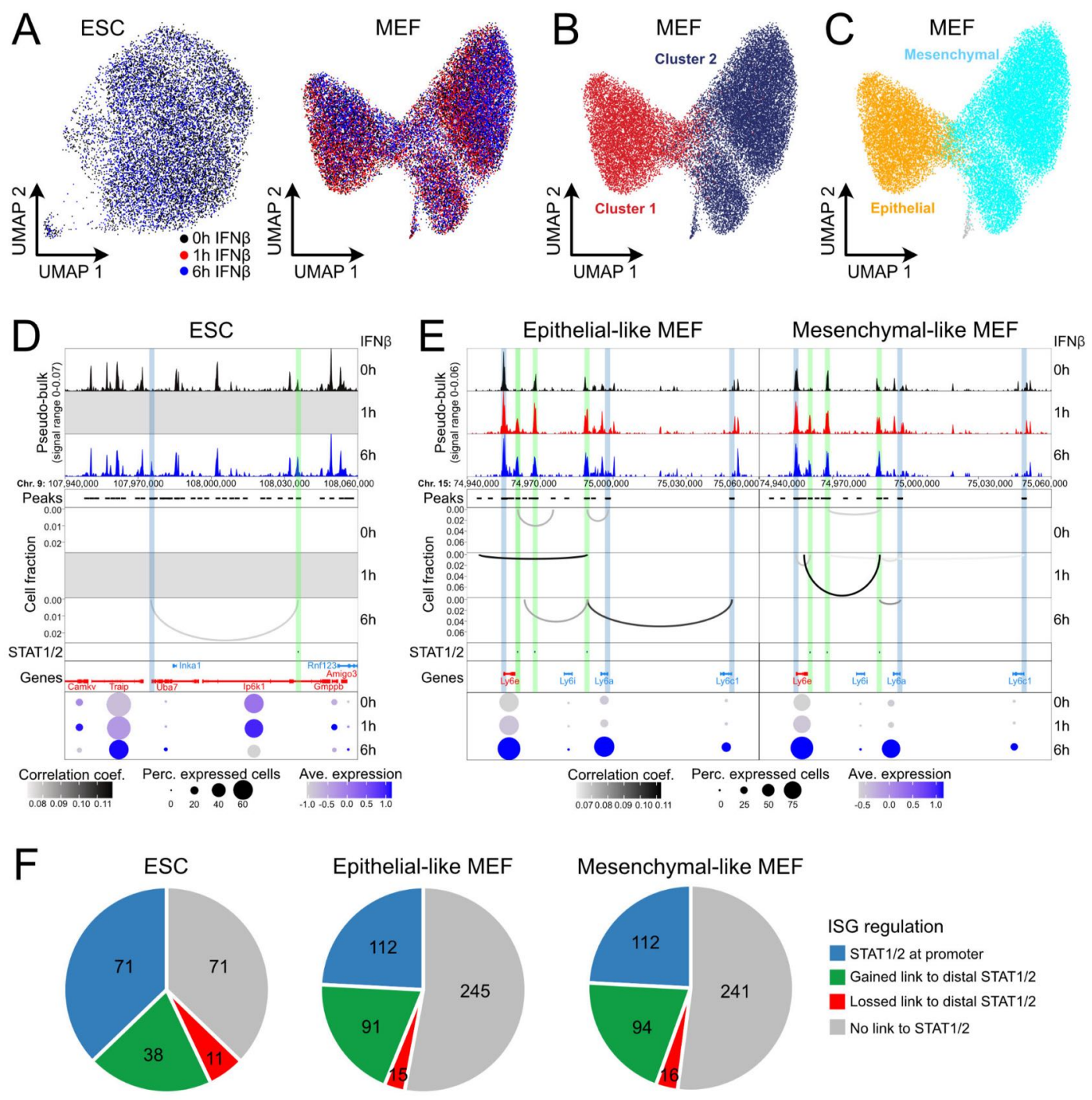

ISG regulation

STAT $1 / 2$ at promoter

Gained link to distal STAT1/2

Lossed link to distal STAT1/2

No link to STAT1/2

G

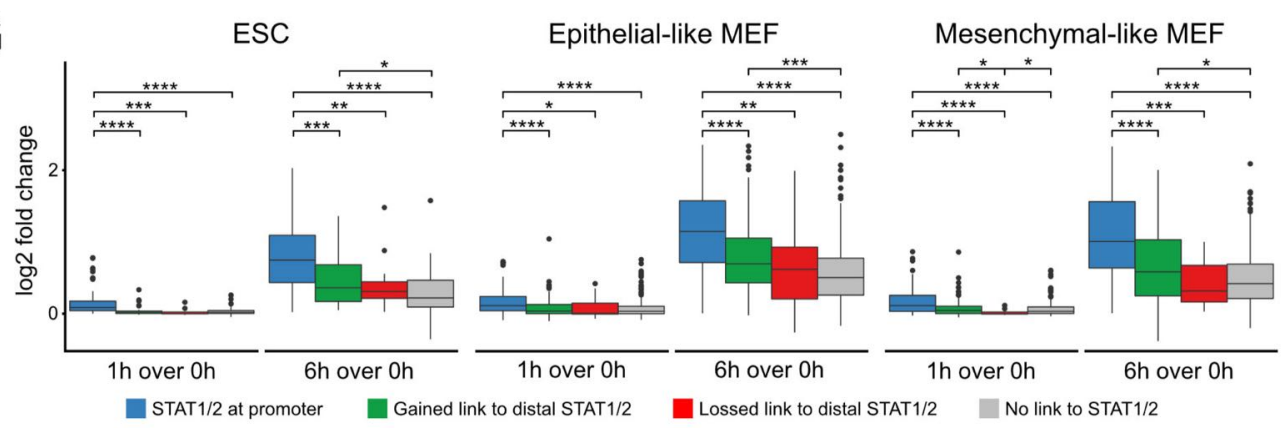

Figure 4. Regulation of ISG expression by distal STAT1/2 binding. (A) Single-cell embedding of chromatin accessibility in ESCs and MEFs with coloring according to treatment. (B) Same as panel A for MEFs with coloring according to clusters predicted by ArchR/Seurat. (C) Same as panel B with coloring according to MEF subtypes according to intregrated scRNA-seq data. (D) Co-accessibility maps before in ESCs of a region around the Uba7 ISG. Top: Browser tracks of pseudo-bulk chromatin accessibility from single cells. Middle: Co-accessible links between the indicated intronic STAT1/2 site and other genomic loci. Bottom: Gene expression levels from scRNA-seq. Experimentally identified ISG promoters and STAT1/2 binding sites were marked by blue and green vertical bars, respectively. Transcription from the Inka1 and the Rnf123 gene was not detected. (E) Same as panel D but for three intergenic STAT1/2 binding sites in the Ly6 ISG cluster in MEFs. (F) ISG regulation by STAT1/2 binding in ESCs (left), epithelial-like (mid), and mesenchymal-like MEFs (right). (G) Expression changes of ISGs for the different STAT1/2 dependent regulation types in ESCs (left), epithelial-like (mid), and mesenchymal-like MEFs (right). 
As an exemplary result, induction of the Uba7 ISG by STAT1/2 binding to a putative distal enhancer in ESCs is depicted in Fig. 4D. The IFN $\beta$-induced co-accessible link between the STAT1/2 bound enhancer candidate and the Uba7 promoter was associated with an increase in Uba7 expression in scRNA-seq. Uba7 was previously identified by bulk RNA-seq data as an ISG (Fig. 1B). Another example of ISG regulation by STAT1/2 binding to distal putative enhancers was the gene cluster of the Ly6 family in MEFs (Fig. 4E). In the gene cluster, expression of ISGs Ly6e, Ly6a and Ly6c1 increased with IFN $\beta$ treatment. The promoter of ISG $L y 6 e$ was highly accessible before and after IFN $\beta$ treatment while $\angle y 6 a$ and $\angle y 6 c 1$ promoters remained in relatively lowly accessible states. In contrast, the three intergenic STAT1/2 binding sites in this genomic region opened up strongly upon IFN $\beta$ treatment. Multiple co-accessible links between the intergenic STAT1/2 sites and ISGs were detected, either directly to the $L y 6$ promoters or indirectly to their gene bodies or proximal regions. These involved the formation of new links between the potential enhancer cluster and the Ly6a and Ly6c1 promoters as well as the loss of links present at the $0 \mathrm{~h}$ time point. With this co-accessibility analysis, we were able to link roughly $25 \%$ of ISGs without STAT1/2 promoter binding to a distal STAT1/2 binding event (Fig. 4F) (ESCs, 38 ISGs; epithelial-like MEFs, 91 ISGs; mesenchymal-like MEFs, 94 ISGs) (Supplementary Data Set 3). Interestingly, we also observed a loss of existing coaccessible links between ISGs and distal STAT1/2 sites at several loci (ESCs, 11 ISGs; epitheliallike MEFs, 15 ISGs; mesenchymal-like MEFs, 16 ISGs), which points to larger changes of the 3D chromatin organization during activation that could involve the resolution of inhibitory interactions.

\section{Binding of STAT1/2 to distal sites efficiently induces target ISG expression}

Next, we investigated the expression induction for the differently regulated ISG categories after $1 \mathrm{~h}$ and $6 \mathrm{~h}$ of IFN $\beta$ treatment over unstimulated control cells and found similar patterns for ESCs and both MEF subtypes (Fig. 4G). After $1 \mathrm{~h}$ of IFN $\beta$ treatment some induction was observed for all cell types and ISG categories. ISGs with a STAT1/2 site at their promoter showed the strongest expression upregulation after $6 \mathrm{~h}$ of IFN $\beta$ treatment, which was significantly stronger than expression induction in all other ISG regulation categories. Additionally, ISGs that gained a co-accessible link to a distal STAT1/2 site showed a significantly stronger expression induction after $6 \mathrm{~h}$ of IFN $\beta$ treatment compared to ISGs without any link to STAT1/2. Moreover, the ISGs with a loss of a preexisting link to a distal STAT1/2 site upon IFN $\beta$ treatment showed a significantly lower gene expression level before IFN $\beta$ treatment $(0 h)$ in ESCs and mesenchymal-like MEFs (Supplementary Fig. 4D). In summary, the scATAC-seq data allowed us to distinguish different mechanisms by which STAT1/2 induces ISG expression from distal sites. It suggests that STAT1/2 induced ISG expression from distal enhancers in addition to binding directly at their promoters. Moreover, our analysis suggests that the loss of pre-existing links during STAT1/2 binding could be associated with the removal of inhibitory interactions.

\section{Five different chromatin states of STAT1/2 binding sites can be distinguished}

The overlap of STAT1/2 peaks from ESCs and MEFs revealed 92 shared binding sites mostly at promoters (70/92). The 116 ESC-specific and 184 MEF-specific sites were predominantly at nonpromoter loci (100/116 and 118/184) (Supplementary Fig. 5A). We reasoned that the cell type specific STAT1/2 binding was dependent on the chromatin context. 


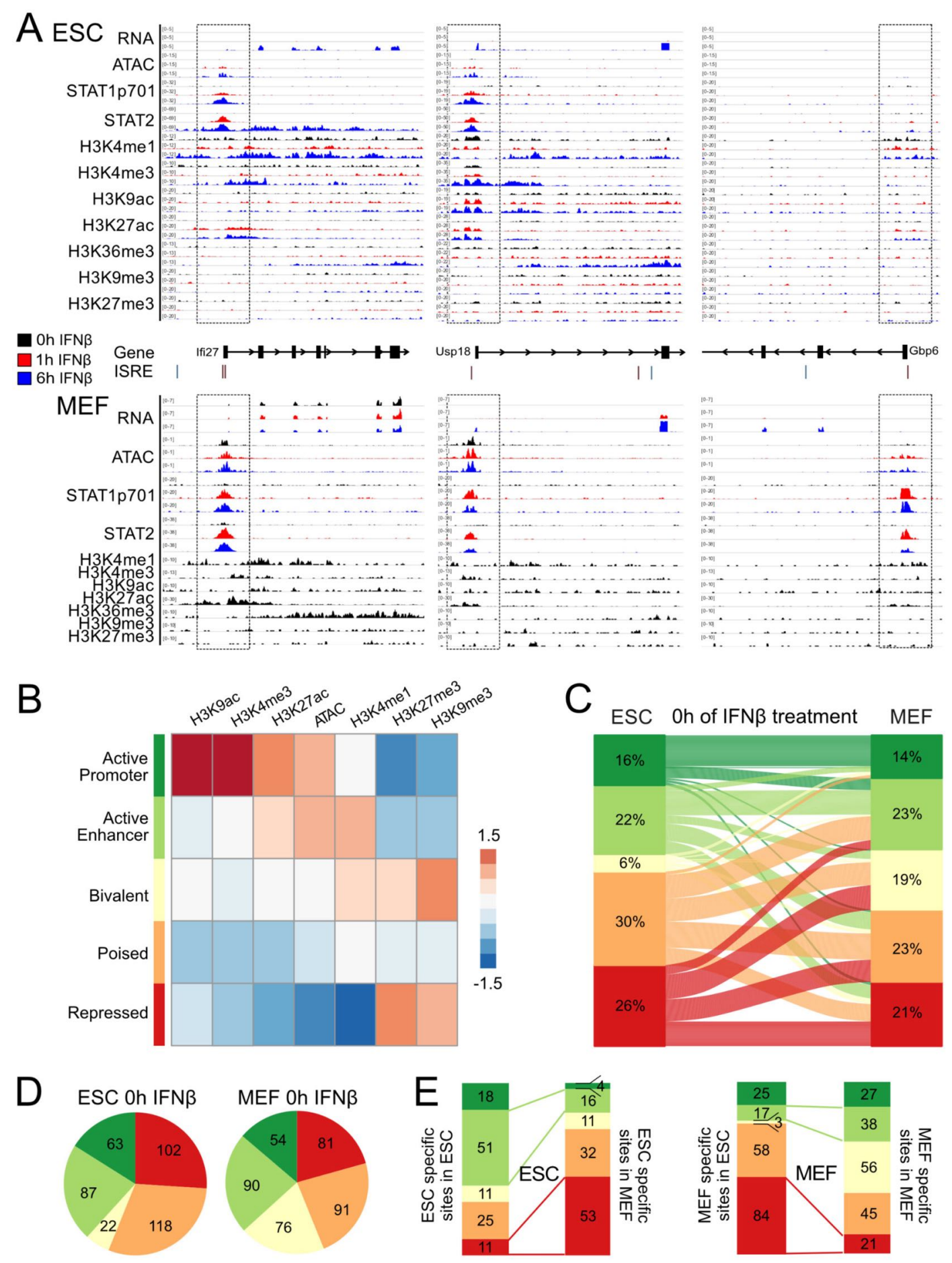

Figure 5. Contribution of chromatin features to STAT1/2 binding. (A) Genomic regions around the ISGs Ifi27, Usp18 and Gbp6 in ESCs (top) and MEFs (bottom) with the different sequencing readouts and the promoter regions marked by boxes. Gene annotation was based on ENSEMBL and the positions of the DNA binding motif IRSE were extracted from the HOMER database. Each browser track shows one representative biological replicate. (B) Heatmap of unsupervised k-means clustering of histone modifications and ATAC data at 392 STAT1/2 binding sites. The indicated five main chromatin states were identified. Data from unstimulated ESCs and MEFs as well as ESCs, at $1 \mathrm{~h}$ and $6 \mathrm{~h}$ IFN $\beta$ treatment were used. (C) Chromatin state transitions between untreated ESCs and MEFs at STAT1/2 binding sites based on the data in panel B and corresponding coloring of the five different chromatin states. (D) Absolute numbers of STAT1/2 binding sites according to chromatin states in unstimulated ESCs and MEFs. (E) Distribution of 116 ESC-specific and 184 MEF-specific STAT1/2 binding sites according to chromatin state. 
Accordingly, we mapped six histone modifications (H3K4me1, H3K4me3, H3K9ac, H3K27ac, $\mathrm{H} 3 \mathrm{~K} 9 \mathrm{me} 3$ and $\mathrm{H} 3 \mathrm{~K} 27 \mathrm{me} 3$ ) by ChIP-seq as well as chromatin accessibility by ATAC-seq. Exemplary regions for ESCs and MEFs were shown (Fig. 5A). STAT1/2 binding at the Usp18 promoter induced the gene in both cell types from a transcriptionally repressed to an active state. In contrast, Ifi27 induction was apparent only in ESCs as compared to a constitutively active state in MEFs while Gbp6 became active in MEFs and remained silent in ESCs. Of note, several additional ISRE motifs did not display STAT1/2 binding illustrating the requirement for a permissive chromatin state (Fig. 5A). To reveal chromatin features that are linked to STAT1/2 binding, normalized read counts in a window of $\pm 1 \mathrm{~kb}$ around the peak center were computed for the different readouts (Supplementary Fig. 5A). These data were then subjected to unsupervised k-means clustering (Fig. 5B, Supplementary Fig. 5B, C). Five main clusters emerged that were annotated based on the combination of enriched chromatin features (Fig. 5B): (i) "Active Promoter" was enriched for H3K4me3, H3K9ac and H3K27ac (Ernst et al, 2011). (ii) "Active Enhancer" was marked by H3K4me1 and H3K27ac (Creyghton et al, 2010). (iii) The "Bivalent" state carried active marks like H3K4me3 and repressive marks like H3K27me3 at the same time (Bernstein et al, 2006). (iv) The "Poised" state showed only H3K4me1 (Creyghton et al, 2010). (v) "Repressed" was marked by enrichment of H3K9me3 or H3K27me3 (Lehnertz et al, 2003; Morey \& Helin, 2010).

\section{STAT1/2 binding is directed by chromatin accessibility and specific histone marks}

Next chromatin states at STAT1/2 sites in ESCs and MEFs and their changes were analyzed (Fig. 5C-E). The most pronounced chromatin state transitions between cell types occurred from the "Poised" and "Repressed" states in ESCs to the "Active Enhancer", "Bivalent" and "Poised" states in MEFs (Fig. 5C). The 116 ESC-specific sites displayed a 3 to 4-fold loss of the "Active Promoter" and "Active Enhancer" states and an approximately 5-fold increase of the "Repressed" state when their chromatin state was evaluated in MEFs (Fig. 5E). Corresponding changes of the "Active Enhancer" and "Repressed" states were also found for MEF-specific sites in ESCs and MEFs. The fraction of MEF-specific STAT1/2 sites in the "Active Promoter" state remained mostly unchanged between cell types, while the number of sites in the "Bivalent" state strongly increased from 3 to 56 sites (Fig. $5 \mathrm{E})$. We conclude that the main changes that determine the cell type specific binding of STAT1/2 occurred between the "Repressed" state (H3K9me3 and H3K27me3) and "Active Enhancer" and "Bivalent" states that both are enriched in the H3K4me1 and H3K27ac modifications. Accordingly, the increased number of ISGs detected in MEFs appears to be related to the more frequent activation of ISRE containing enhancer elements.

To further dissect the relation between chromatin signals in the uninduced state and STAT1/2 binding upon induction we computed their correlations. Normalized read counts of a given chromatin feature before induction were plotted against STAT1/2 binding as represented by the average signal of STAT1 and STAT2 at $1 \mathrm{~h}$ of IFN $\beta$ treatment at the same locus (Fig. 6A). These plots visualized the differences between ESC-specific (black) and MEF-specific (red) binding sites for specific chromatin features. The $p$-value and correlation coefficient $R$ of a given mark with STAT1/2 binding are plotted in Fig. 6B. ATAC (ESC, $R=0.42$; MEF, $R=0.53$ ), H3K4me1 (ESC, $R=0.45$; MEF, $R=$ 0.43 ) and H3K27ac (ESC, $R=0.41$; MEF, $R=0.63$ ) were the most strongly positively correlated marks, while H3K27me3 (ESC, $R=-0.23$; MEF, $R=-0.39$ ) was anticorrelated with STAT1/2 binding. For the repressive $\mathrm{H} 3 \mathrm{~K} 9 \mathrm{me} 3$ mark the correlation was negative for ESCs $(R=-0.26)$ and slightly 
positive for MEFs $(R=0.08)$ pointing to a more complex relation. We concluded that a pre-existing active chromatin state (open chromatin, H3K4me1 and H3K27ac) promoted STAT1/2 binding while chromatin loci marked by the H3K27me3 impeded this interaction (Fig. 6C).

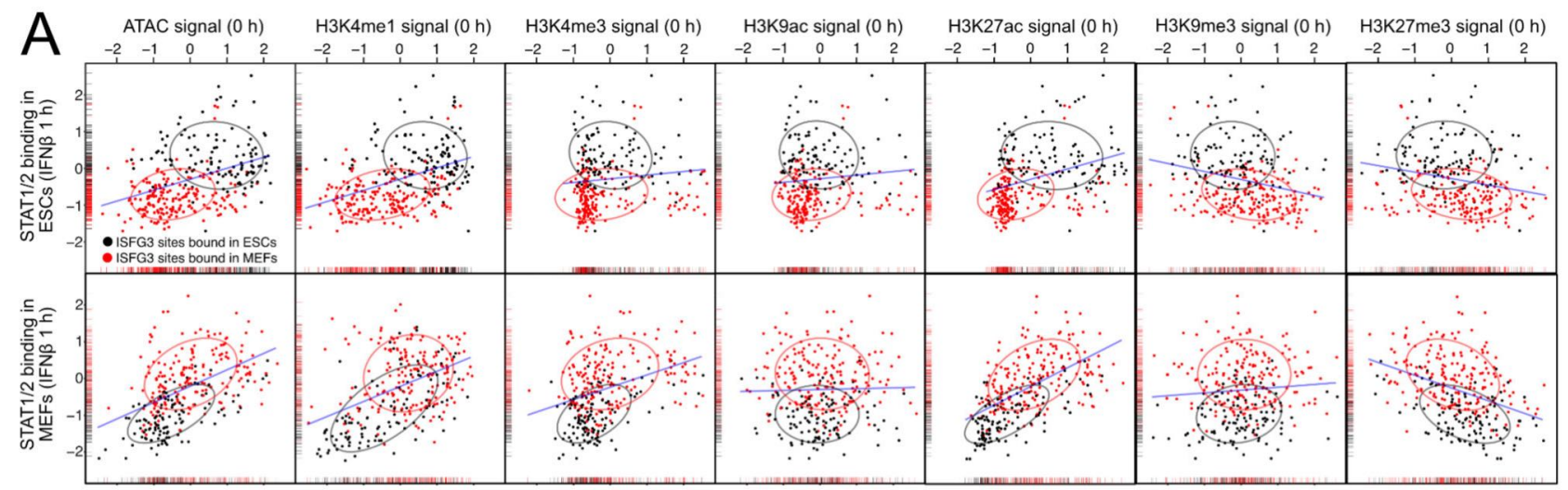

B

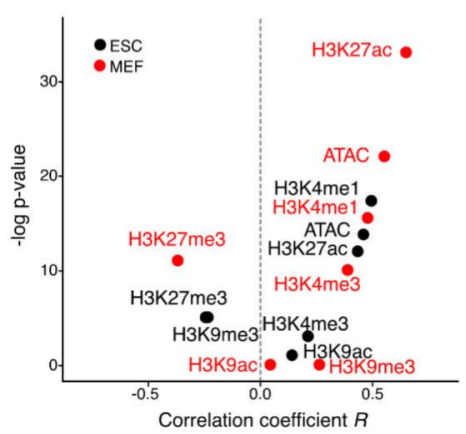

C

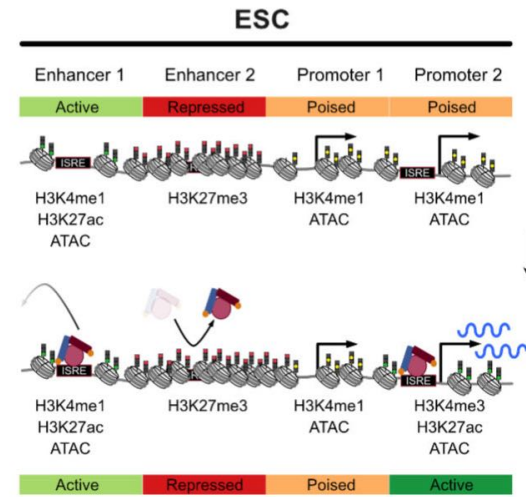

MEF

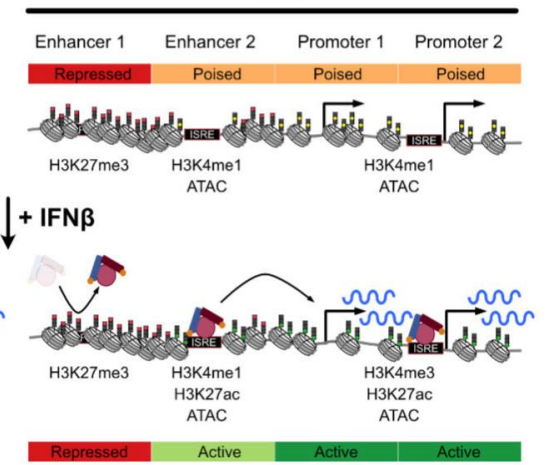

Figure 6. Correlation of STAT1/2 binding with pre-existing chromatin environment. (A) Correlation between STAT1/2 binding after $1 \mathrm{~h}$ of IFN $\beta$ treatment and pre-existing chromatin features before IFN $\beta$ treatment. The STAT1/2 binding signal was computed as the average signal of STAT1 and STAT2 after $1 \mathrm{~h}$ IFN $\beta$ treatment in ESCs (top) and MEFs (bottom). The chromatin features were quantified by counting the normalized read counts at the STAT1/2 binding sites before induction. ESC-specific STAT1/2 binding sites are shown in black and MEF-specific ones in red. Ellipses indicate the area, in which $75 \%$ of all data points are located. Density distributions are shown along the $x$ - and $y$-axis. The blue line shows the linear regression of the combined set of ESC-and MEF-specific STAT1/2 binding sites. (B) Correlation between STAT1/2 binding and chromatin features determined for the data in panel A in ESCs (black) and MEFs (red). (C) Scheme of cell type specific ISG induction via STAT1/2 binding. The most prominent differences between cell types are depicted. Enhancers as well as promoters direct STAT1/2 binding in dependence of cell type specific chromatin states. Some ISGs have accessible ISRE motifs at their promoter and can directly be activated by STAT1/2 binding while a repressive chromatin state impedes binding at other promoters. The same applies to ISGs that lack an ISRE at the promoter but are activated by STAT1/2 binding to enhancers that induces transcription of a target ISG from a distal site.

\section{Discussion}

We compared ESCs to in vitro differentiated NPCs as well as MEFs from the same mouse strain to reveal mechanisms that govern the cell type specific response to IFN $\beta$ in a genome wide manner. In total 513 ISGs were identified, in line with previous studies that reported between 200 to 1,000 
upregulated genes in different cellular systems (de Veer et al, 2001; Der et al, 1998; Mostafavi et al, 2016). Our results corroborate the finding that ESCs show an attenuated response to IFN $\beta$ (D'Angelo et al, 2016; Gonzalez-Navajas et al, 2012; Guo, 2017; Guo et al, 2015; Wang et al, 2014; Wang et al, 2013; Whyatt et al, 1993). This stem cell specific feature appears to be compensated by a constitutive expression of some ISGs in human stem cells (Wu et al, 2018) as well as the presence of an antiviral RNA interference based system in mouse ESCs (Maillard et al, 2013; Poirier et al, 2021). A previous RT-qPCR analysis of selected components of the IFN signaling pathway in ESCs identified a significant downregulation of the IFNa/ $\beta$ receptor Ifnar1 while Stat2, Tyk2 and Irf9 were upregulated as compared to a MEF cell line (Wang et al, 2014). Based on our differential RNA-seq maps of the unstimulated cell types we confirm the downregulation of Ifnar1 in ESCs while the differences for Stat2, Tyk2 and Irf9 were above the $p<0.01$ significance level. We additionally detected a strong downregulation of Ifnar2, the Ifngr1/2 and the Jak1/2 kinases in ESCs relative to NPCs and MEFs. Furthermore, both STAT1 and STAT2 as well as phosphorylated STAT1 were more abundant in MEFs than in ESCs at the protein level after induction. Thus, a globally reduced IFN $\beta$ response could be assigned to lower levels of key components of the IFN signaling pathway in ESCs.

Previous studies on STAT1/2 binding reported 6,703 STAT2 peaks for IFNa treated B cells (Mostafavi et al, 2016), and 41,582 (IFNY-stimulated) and 11,004 (unstimulated) STAT1 binding sites in HeLa S3 cells (Robertson et al, 2007). The specificity of STAT peak assignment in these previous studies appears to be moderate. A fraction of $46 \%$ of the STAT2 peaks displayed a $>2$-fold increase upon IFNa treatment (Mostafavi et al, 2016), while a 2-5 fold enrichment of GAS and ISRE sequences in the STAT1 peaks was present (Robertson et al, 2007). Our identification of STAT1 p701 and STAT2 binding sites was more stringent and displayed an at least 4-fold STAT enrichment upon induction. In addition, $80-90 \%$ of the sites carried a STAT- or IRF-family binding site sequence motif with a more than 10-fold higher frequency than found in the background sequences. It is further noted that we did not detect STAT2 ChIP-seq peaks before the IFN $\beta$ stimulus. Thus, an activity of unphosphorylated STAT2-IRF9 for basal gene expression of ISGs as reported in (Blaszczyk et al, 2015) was not apparent in the STAT2 binding maps recorded here.

The main ISG activation sites in our system had STAT1 and STAT2 bound simultaneously most likely within the ISGF3 complex that additionally involves IRF9 and in line with previous findings (AuYeung et al, 2013; Lee \& Young, 2013; Singh et al, 2014). This assignment was confirmed by the binding motif analysis that yielded an enrichment of IRF motifs in $80-90 \%$ of the 392 STAT1/2 peaks. The number of sites that had only STAT $1_{\text {p701 }}$ or STAT2 bound was 1,037 and 323 , respectively (Supplementary Table S2). STAT1 homodimers can also act as activators of type I IFN response (Stanifer et al, 2019; Stark \& Darnell, 2012). However, the promoters that only had STAT1 p701 $_{\text {bound }}$ showed no enrichment for ISGs in our data set. An additional minor contribution to ISG activation arose from binding of STAT2 in the absence of STAT1 at ISG promoters, which is in line with the observation that the STAT2-IRF9 complex has some activation capacity without STAT1 (Platanitis et al, 2019).

Interestingly, more than $2 / 3$ of the STAT1/2 peaks were located at intergenic or intronic regions and thus represent potential enhancer elements that could drive ISG activation. The target ISGs of these putative enhancers did not appear to be those that were in closed genomic distance. We identified 
links between ISG promoters and distal STAT1/2 binding sites by applying a co-accessibility analysis of the scATAC-seq data (Granja et al, 2021; Mallm et al, 2019). This approach exploited the accessibility information obtained from thousands of single cells to compute pair-wise correlations of sites with bound STAT1/2 and ISG promoters. These correlations could originate from direct spatial contacts or other mechanisms of enhancer-promoter communication (Karr et al, 2022). With this approach we were able to link $25 \%$ of ISGs lacking STAT1/2 at the promoter to a distal STAT1/2 binding site that is likely to represent an enhancer that activates this gene. In addition, our data suggest that IFN $\beta$ induction and STAT1/2 binding could also involve the removal of pre-existing inhibitory links between ISGs and distal regulatory regions. The latter process might be related to the loss of long-range interactions observed during induction of differentiation in mouse ESCs (Feldmann et al, 2020). Furthermore, a recent study describes the reorganization of the 3D genome around ISG loci upon both IFN $\beta$ and IFNy treatment, which involves loop formation, nucleosome remodeling and an increase of DNA accessibility (Platanitis et al, 2021). Thus, it is emerging that a reorganization of long-range chromatin interactions represents an important part of IFN mediated gene induction.

According to the HOMER data base (Heinz et al, 2010), ISREs are and are found at 134,069 loci in the mouse genome. According to our ChIP-seq analysis a much lower number of 392 ISREs had STAT1/2 bound. This large difference led us to characterize their chromatin environment as a determinant of STAT binding via a genome-wide correlation analysis. We find that a repressive chromatin conformation marked by H3K27me3 renders ISREs less accessible to STAT1/2 binding. In contrast, H3K4me1 and H3K27ac as well as an open chromatin state detected by ATAC were associated with sites permissive for STAT1/2 binding. These results are in line with a previous study that compared histone modifications at 18 ISREs (Testoni et al, 2011). In the latter data set, 6 out of 9 ISREs at activated promoters showed some enrichment for H3K4me1 before induction with IFNa. It is noted that $\mathrm{H} 3 \mathrm{~K} 4 \mathrm{me} 1$ has been related to targeting the BAF (SWI/SNF) chromatin remodeler to chromatin, which interacts with STAT1 1 p701 and STAT2 via its BRG1 component. Accordingly, this histone modification could promote chromatin opening and subsequent STAT1/2 binding (Christova et al, 2007; Huang et al, 2002; Local et al, 2018).

\section{Conclusions}

Our integrated multi-omics data set provides insight into the interplay between the IFN $\beta$ mediated activation of ISGs, STAT binding and chromatin features. It revealed a number of links that could be exploited to modulate the IFN response during virus infection or therapeutic intervention in cancer (Borden, 2019; Hoffmann et al, 2015). Numerous so called "epigenetic drugs" that inhibit enzymes setting or removing histone acetylation and methylation are already used in anti-cancer therapy (Cheng et al, 2019; Mohammad et al, 2019). In the light of our study, the resulting perturbances of chromatin features are also likely to affect IFN response. For example, histone deacetylase (HDAC) inhibitors that result in the hyper-acetylation of histones and render chromatin more accessible could enhance STAT1/2 binding to otherwise occluded ISREs (Cusack et al, 2020; Gorisch et al, 2005; Shogren-Knaak et al, 2006; Wang \& Hayes, 2008). At the same time, however, these drugs also affect the acetylation state of protein factors involved in IFN mediated signaling like the acetylation and activity of the STAT1/2 complex itself (Tang et al, 2007). Accordingly, HDACs have been shown 
to both repress or enhance IFN response in a complex manner (Au-Yeung \& Horvath, 2018; Lu et al, 2019). Thus, changing STAT1/2 binding patterns more specifically would require a targeted approach beyond global inhibition/activation of epigenetic modifiers like HDACs. This could be achieved, for example, by using more selective drugs (Cheng et al, 2019; Mohammad et al, 2019) or dCas 9 mediated changes of ISRE chromatin states at promoters and enhancers by targeted binding of activators that sets or remove H3K27ac, H3K4me1 or H3K27me3 (Erdel et al, 2020; Frank et al, 2021; Li et al, 2020). In this manner, ISG activation patterns could be changed to modulate the cell type specific antiviral response.

\section{Materials and methods}

\section{Cell culture work and IFN $\beta$ treatment}

Mouse 129/Ola ESCs, NPCs differentiated in vitro from ESCs and MEFs were cultured at $37^{\circ} \mathrm{C}$ with $5 \% \mathrm{CO}_{2}$ and routinely checked for the absence of mycoplasma contaminations as described previously (Bibel et al, 2007; Mallm et al, 2020; Teif et al, 2012). IFN $\beta$ was prepared from a BHK cell line over-expressing IFN $\beta$ and grown with DMEM supplemented with $10 \%$ FCS, $1 \%$ L-glutamine and $1 \%$ penicillin/streptomycin. After growing the cells in the same medium but with $2 \%$ FCS for 24 $\mathrm{h}$ the IFN $\beta$ containing medium was passed through a $0.45 \mu \mathrm{m}$ sterile filter and stored in aliquots at $-80^{\circ} \mathrm{C}$. The resulting IFN $\beta$ stock was calibrated against a commercial IFN $\beta$ preparation (Sigma) by using a Mx2-luciferase reporter cell line (Schwerk et al, 2013). A stock concentration of $16.6 \mathrm{U} / \mu \mathrm{l}$ was calculated. For induction, cells were treated with IFN $\beta$ at a concentration of $500 \mathrm{U} / \mathrm{ml}$ for 1 hour or 6 hours.

\section{Western blots}

Western blot samples were prepared by collecting cells directly out of cell culture. Cells were transferred into $1.5 \mathrm{ml}$ tubes, washed once with PBS and counted. A $50 \mu \mathrm{l}$ volume of pre-prepared RIPA buffer (150 mM NaCl, 1 \% NP40, 50 mM Tris-HCl, pH 8.0, $0.5 \%$ sodium deoxycholate, $0.1 \%$ sodium dodecyl sulfate) were added per 0.5 million cells in suspension. The mixes were incubated for $60 \mathrm{~min}$ on ice, spun down at max speed at $4{ }^{\circ} \mathrm{C}$ for $30 \mathrm{~min}$. Supernatant was transferred to a fresh tube and stored at $-20^{\circ} \mathrm{C}$. Gels were blotted on LF PVDF membranes using the trans-blot turbo transfer system (Bio-Rad) and blocked with $5 \%$ BSA in Tris-buffered saline supplemented with $0.1 \%$ Tween 20 detergent (TBST) at room temperature for $1 \mathrm{~h}$. The primary antibodies were diluted according to manufacturer's recommendations in $5 \%$ BSA and incubated at $4{ }^{\circ} \mathrm{C}$. On the following day, the membrane was washed three times with TBST buffer at room temperature for 5 min under agitation and incubated with secondary anti-HRP antibody (normally 1:5000 diluted in $5 \%$ BSA) at room temperature for $1 \mathrm{~h}$, washed three times with TBST, incubated with clarity western ECL substrate for $5 \mathrm{~min}$ and imaged. The antibodies used are listed in Supplementary Table S4.

\section{Bulk RNA-seq}

Cells were seeded on a 6-well plate. Two (ESCs and MEFs) or five (NPCs) days after plating, cells were washed two times with PBS. Then $500 \mu \mathrm{LBP}$ was added and cells were stored at $-80^{\circ} \mathrm{C}$. RNA was isolated with the Nucleospin RNA kit (Macherey-Nagel) according to the manufacturer's protocol. The elution step was done two times with $30 \mu \mathrm{l}$ RNase-free water within the same tube. 
Concentrations were measured by Qubit RNA HS assay kit and the quality of RNA was analyzed on Tapestation D5000 HS (Agilent). Removal of rRNAs from isolated samples of IFN $\beta$ stimulated ESCs and MEFs was done following the protocol of Ribo-Zero rRNA removal kit. An input of $5 \mu \mathrm{g}$ total RNA was used and the depleted RNAs were eluted in $30 \mu$ l Rnase-free water supplemented with $1 \mu \mathrm{l}$

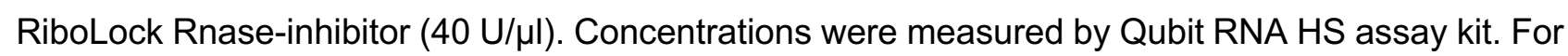
NPCs, RNA samples were treated with Dnase at $37{ }^{\circ} \mathrm{C}$ for $30 \mathrm{~min}$ and purified by ethanol precipitation. Concentrations were measured by Qubit RNA HS assay kit and 750 ng of Dnasetreated RNA was used for rRNA depletion by NEB Next rRNA depletion kit (Human/Mouse/Rat). The depletion was performed based on the manufacturer's protocol. Samples were purified with RNA Clean XP beads (Beckman) with a 2.2x ratio and finally eluted in $8 \mu$ nuclease-free water. Purified rRNA-depleted RNA samples of ESCs, MEFs and NPCs were used to prepare NGS libraries based on the NEB Next Ultra II directional RNA library preparation kit from Illumina. As default, 50 ng of rRNA-depleted RNA was used as input. For less concentrated samples, $10 \mathrm{ng}$ were used. The RIN value of all samples were above 7 and therefore the mixes were incubated at $94{ }^{\circ} \mathrm{C}$ for $15 \mathrm{~min}$. Further, a 5-fold NEB Next adaptor dilution was used as default at the adaptor ligation step. For lower concentrated samples, a 25-fold dilution was used. All samples were dual-barcoded with unique i5 and i 7 primers. For $50 \mathrm{ng}$ samples a total of nine cycles and for $10 \mathrm{ng}$ samples eleven cycles were performed during the PCR enrichment of the adaptor ligation DNA step. Samples were measured with Qubit dsDNA HS assay kit and the fragment size was determined with a Tapestation D5000. In total, four replicates of ESCs, two replicates of MEFs and four replicates of NPCs treated with IFN $\beta$ for $0 \mathrm{~h}, 1 \mathrm{~h}$ and $6 \mathrm{~h}$ were acquired. The corresponding RNA-seq libraries were 50-bp single-end sequenced on the HiSeq 4000 System (Illumina) with at least 50 million reads per sample. Sequencing of RNA, as well as that of all other sequencing readouts, was done at the DKFZ Genomics and Proteomics Core Facility.

\section{ChIP-seq of STAT1 $1_{\text {p } 01}$ and STAT2}

STAT1 1 p701 and STAT2 ChIPs were performed with the ChIP enzymatic chromatin IP kit from Cell Signaling Technology according to the manufacturer's protocol. Around $4 \times 10^{6}$ cells per sample were used as input for the ChIPs. The Mnase digestion step was not used for ESCs and MEFs after formaldehyde fixation. Chromatin fragmentation was done with the Epishear probe sonicator (Active Motif) at $4{ }^{\circ} \mathrm{C}$ with $30 \mathrm{~s}$ long on and off cycles and $50 \%$ amplitude. For ESCs and MEFs ten cycles of chromatin fragmentation were performed to yield an average fragment size of around $150 \mathrm{bp}$. The immunoprecipitation was conducted with $10 \mu \mathrm{g}$ of chromatin in a total volume of $500 \mu \mathrm{l}$ and addition of antibodies (Supplementary Table S3). The sequencing libraries were prepared using the NEB Next Ultra II DNA library preparation kit for Illumina with $40 \mu \mathrm{l} \mathrm{ChIP} \mathrm{sample} \mathrm{and} \mathrm{added} 10 \mu 10 \mathrm{mM}$ Tris- $\mathrm{HCl} \mathrm{pH}$ 8.0. For the input reaction, a 1:10 dilution was made and from this dilution $4 \mu \mathrm{g}$ chromatin were used and filled up with $1 \times 10 \mathrm{mM}$ Tris- $\mathrm{HCl} \mathrm{pH} 8.0$ to a total volume of $50 \mu \mathrm{l}$. Concentrations were measured by Qubit dsDNA HS assay kit and fragment distribution was analyzed on a Tapestation D5000. The libraries were sequenced as described above for RNA-seq.

\section{ChIP-seq of histone modifications}

ESCs were cultured in $150 \mathrm{~mm}$ dishes and treated with IFN $\beta$ for $0 \mathrm{~h}, 1 \mathrm{~h}$ or $6 \mathrm{~h}$. Media was removed and cells were detached with Accutase, washed with PBS supplemented with PMSF at $0.5 \mathrm{mM}$ 
concentration and crosslinked with $1 \%$ formaldehyde ( $1 \mathrm{ml} 16 \%$ formaldehyde with $15 \mathrm{ml} \mathrm{PBS}$ ) for $10 \mathrm{~min}$ at room temperature. $125 \mathrm{mM}$ glycine was added to neutralize the formaldehyde and rotated at room temperature for $5 \mathrm{~min}$. Afterwards, samples were washed three times with PBS/100 mM PMSF and cell pellets were resuspended in $10 \mathrm{ml}$ swelling Buffer $(25 \mathrm{mM}$ Hepes pH 7.8, $1 \mathrm{mM}$ $\mathrm{MgCl}_{2}, 10 \mathrm{mM} \mathrm{KCl}, 0.1 \% \mathrm{NP}-40,1 \mathrm{mM} \mathrm{DTT}, 0.5 \mathrm{mM}$ PMSF). A $10 \mathrm{~min}$ incubation step on ice and a centrifugation step at $2000 \mathrm{rpm}$ for $5 \mathrm{~min}$ at $4{ }^{\circ} \mathrm{C}$ was performed. $4 \times 10^{7}$ cells were resuspended in $100 \mu \mathrm{l}$ Mnase Buffer and $40 \mathrm{U}$ Mnase was added. After an incubation step at $37^{\circ} \mathrm{C}$ for $15 \mathrm{~min}$, $100 \mu \mathrm{l}$ of $10 \mathrm{x}$ sonication buffer and $800 \mu \mathrm{l}$ water were added. Samples were incubated on ice for $5 \mathrm{~min}$, transferred into $12 \times 24 \mathrm{~mm}$ tubes and sonicated for $15 \mathrm{~min}$ (burst 200; cycle $20 \%$; intensity 8 ) on a Covaris sonicator. A centrifugation step was performed at $13,000 \mathrm{rpm}$ and $4{ }^{\circ} \mathrm{C}$ for $15 \mathrm{~min}$. The supernatant was transferred into fresh tubes and chromatin was snap frozen with liquid nitrogen and store at $-80^{\circ} \mathrm{C}$. A quality check of reverse cross-linked samples was performed and yielded a fragment size of around $150 \mathrm{bp}$ for the sheared chromatin. Pre-equilibrated $25 \mu \mathrm{l}$ protein $\mathrm{G}$ beads were used per sample at room temperature for $10 \mathrm{~min}$ in sonication buffer $(10 \mathrm{mM}$ Tris $\mathrm{pH} 8.0$, $200 \mathrm{mM} \mathrm{NaCl}, 1 \mathrm{mM}$ EDTA, 0.1 \% Na-deoxycholate, $0.5 \%$ n-lauroylsarcosine, $0.5 \mathrm{mM}$ PMSF). A sample precleaning step was performed by adding $25 \mu$ l Protein $\mathrm{G}$ beads with $4 \mu \mathrm{g}$ IgG antibody (rabbit or mouse) to chromatin and incubated rotating at $4{ }^{\circ} \mathrm{C}$ for $2 \mathrm{~h}$. Beads were pelleted, and supernatant was transferred to fresh tubes. Antibodies were added to chromatin samples and incubated at $4{ }^{\circ} \mathrm{C}$ for $2 \mathrm{~h}$. Then, $25 \mu \mathrm{l}$ of pre-equilibrated beads were added to the samples and incubated rotating at $4{ }^{\circ} \mathrm{C} O / \mathrm{N}$. The beads were washed by rotating at $4{ }^{\circ} \mathrm{C}$ for $5 \mathrm{~min}$ with high-salt buffer (50 mM Hepes, pH 7.9, 500 mM NaCl, 1 mM EDTA, $1 \%$ Triton-X-100, $0.1 \%$ Na-deoxycholate, $0.1 \%$ SDS, $0.5 \mathrm{mM}$ PMSF), lithium buffer (20 mM Tris pH 8.0, $1 \mathrm{mM}$ EDTA, $250 \mathrm{mM} \mathrm{LiCl,} 0.5 \%$ NP-40, $0.5 \%$ Na-deoxycholate; $0.5 \mathrm{mM} \mathrm{PMSF}$ ) and 2x with TE-buffer (10 mM Tris pH 8.0, $1 \mathrm{mM}$ EDTA). Each sample was eluted two times with $250 \mu$ l elution buffer ( $50 \mathrm{mM}$ Tris $\mathrm{pH} 8.0,1 \mathrm{mM}$ EDTA, $1 \% \mathrm{SDS}, 50 \mathrm{mM} \mathrm{NaHCO}$ ) at $37^{\circ} \mathrm{C}$ for $15 \mathrm{~min}$ on a shaker. Reverse cross-linking was performed by adding $20 \mu \mathrm{l} \mathrm{M} \mathrm{NaCl}$ and incubation at $65^{\circ} \mathrm{C}$ overnight. $10 \mu \mathrm{EDTA}(0.5 \mathrm{M}), 0.5 \mu \mathrm{l}$ Rnase $\mathrm{A}(10 \mathrm{mg} / \mathrm{ml})$ and $50 \mu \mathrm{l}$ Tris $(1 \mathrm{M}, \mathrm{pH} 6.8)$ was added and incubated at $37^{\circ} \mathrm{C}$ for $30 \mathrm{~min}$. Then, $2 \mu$ l Proteinase $\mathrm{K}(20 \mathrm{mg} / \mathrm{ml})$ was added and incubated at $55^{\circ} \mathrm{C}$ for $2 \mathrm{~h}$. An isopropanol precipitation was performed to purify the DNA. Samples were resuspended in water. Samples were measured with Qubit dsDNA HS assay kit and the fragment size was determined on a Tapestation D5000. Libraries were sequenced as described above for RNA-seq. In ESCs, two replicates for H3K4me1, H3K4me3 and H3K27ac and three replicates for H3K9ac, H3K9me3 and H3K27me3 were sequenced. In MEFs, two replicates of all modifications were sequenced.

\section{Bulk ATAC-seq}

ESCs were plated on 6 well plates and treated for $0 \mathrm{~h}, 1 \mathrm{~h}$ or $6 \mathrm{~h}$ with IFN $\beta$ at $500 \mathrm{U} / \mathrm{ml}$. Cells were detached using accutase, collected and washed with 1XMT-PBS. A total of 50,000 cells were transferred into fresh tubes and centrifuged by $800 \mathrm{~g}$ at $4{ }^{\circ} \mathrm{C}$ for $5 \mathrm{~min}$. For ESCs, the cell pellet was resuspended in $200 \mu$ ATAC lysis buffer (10 mM Tris- $\mathrm{HCl} \mathrm{pH} \mathrm{7.4,} 10 \mathrm{mM} \mathrm{NaCl}_{2}, 3 \mathrm{mM} \mathrm{MgCl}, 0.1 \%$ $\mathrm{NP}-40$ ), incubated at room temperature for $2 \mathrm{~min}$ and centrifuged at $800 \mathrm{~g}$ and $4{ }^{\circ} \mathrm{C}$ for $5 \mathrm{~min}$. Supernatant was discarded and pellets resuspended in $20 \mu \mathrm{l}$ ATAC reaction buffer containing $10 \mu \mathrm{l}$ $2 \mathrm{x}$ transposase buffer and $2.5 \mu \mathrm{l}$ Tn5 enzyme (Illumina). Samples were incubated at $37^{\circ} \mathrm{C}$ for $30 \mathrm{~min}$. Reactions were stopped by adding $5 \mu \mathrm{LDTA}(100 \mathrm{mM})$ in $\mathrm{Tris}-\mathrm{HCl} \mathrm{pH} 8.0$ to final 
concentration of $20 \mathrm{mM}$. For MEFs and NPCs, the cells were directly resuspended in $25 \mu \mathrm{l}$ ATAC reaction buffer with digitonin $\left(9.75 \mu \mathrm{l} \mathrm{H}_{2} \mathrm{O}, 12.5 \mu \mathrm{l} 2 \mathrm{x}\right.$ transposase buffer (Illumina), $0.5 \mu \mathrm{l} 50 \mathrm{x}$ proteinase inhibitor, $2 \mu \mathrm{l}$ Tn5 enzyme (Illumina), $0.25 \mu \mathrm{l} \%$ digitonin) and incubated at $37^{\circ} \mathrm{C}$ for $30 \mathrm{~min}$. The samples were purified with a MinElute PCR purification kit (Qiagen) and eluted in $12 \mu \mathrm{l}$ buffer. After PCR amplifications, sequencing libraries were purified with AMPure beads (Beckman). Concentration was measured with the Qubit dsDNA HS assay kit (ThermoFisher) on a Qubit fluorometer, and size distribution of final library was checked on a Tapestation D5000. Libraries were 50-bp paired-end sequenced on Illumina HiSeq 2000 or 4000 systems with at least 50 million reads per sample. Two replicates for ESCs and NPCs and four for MEFs were sequenced.

\section{Analysis of bulk sequencing data}

For RNA-seq analysis, ribosomal RNAs were removed and raw reads were mapped with STAR (Dobin et al, 2013) to the mm10 mouse reference genome and normalized read counts (transcripts per kilobase million, TPMs) were computed with RSEM (Li \& Dewey, 2011). The differential gene expression analysis between treated and untreated controls was performed using DESeq2 (Love et al, 2014) with $p$-value $<0.05$ and log fold change $>1.5$. For the analysis of ChIP-seq and ATAC-seq data, reads were mapped with Bowtie2 (Langmead \& Salzberg, 2012) to the mm10 mouse reference genome. Duplicates and reads annotated to blacklisted regions (Encode Project Consortium, 2012) as well as mitochondrial reads were removed. Quality control followed the Encode guidelines (https://www.encodeproject.org/data-standards/chip-seq/) and involved the fraction of reads in peaks (FriP) scores, normalized strand coefficients (NSC) and relative strand correlation (RSC) values for each sample. Peak calling was done with MACS2 (Zhang et al, 2008) and for H3K9me3 and H3K27me3 also with SICER (Xu et al, 2014) with p-value threshold of $10^{-5}$. STAT1 1 701 and STAT2 binding sites were identified against the unstimulated controls for $1 \mathrm{~h}$ and $6 \mathrm{~h}$ of IFN $\beta$ treatment from the ChIP-seq data with Diffbind (Ross-Innes et al, 2012) using the consensus peak list and thresholds of FDR $<0.05$ and 4-fold enrichment. Sequence motifs enriched in STAT1, STAT2 and STAT1/2 peaks were identified using HOMER (Heinz et al, 2010). For the analysis of the STAT1/2 chromatin environment, STAT1/2 bound sites in ESCs and MEFs were expanded by $1 \mathrm{~kb}$ up- and downstream. The ChIP- and ATAC-seq signal in these regions was determined from the respective read counts after normalizing for library depth and fragment length and computing enrichments over histone $\mathrm{H} 3$ for histone modifications and IgG for STAT1/2. Replicates of the same samples and time points of IFN $\beta$ stimulation were averaged. The resulting count tables were used as input for the k-means clustering to characterize the chromatin environment at STAT1/2 binding sites.

\section{Single-cell RNA-seq and ATAC-seq}

The scRNA-seq experiments were performed based on the standard protocol for the Chromium single-cell 3' reagent kit v2 (10x Genomics). ESCs and MEFs were treated for $0 \mathrm{~h}, 1 \mathrm{~h}$ or $6 \mathrm{~h}$ with IFN $\beta$. The cDNA amplification was done by running 13 PCR cycles. The samples were eluted again in $35 \mu \mathrm{l} 10 \mathrm{mM}$ Tris- $\mathrm{HCl} \mathrm{pH}$ 8.0. Concentrations of cDNA libraries were measured by Qubit dsDNA $\mathrm{HS}$ assay kit and mean peak sizes of the samples were determined on a Tapestation D5000. Each of the final libraries were paired-end sequenced ( $26 \mathrm{bp}$ and $74 \mathrm{bp}$ ) on one Illumina HiSeq 4000 lane. For scATAC-seq, ESCs and MEFs were treated with IFN $\beta$ for $0 \mathrm{~h}, 1 \mathrm{~h}$ (only for MEFs) or $6 \mathrm{~h}$ and 
libraries were prepared according to the Chromium single-cell ATAC v1.0 protocol (10x Genomics). Two (three for MEFs treated with IFN $\beta$ for $6 \mathrm{~h}$ ) lane replicates per scATAC libraries were paired-end sequenced on an Illumina NovaSeq 6000 system according to the manufacturer's protocol.

\section{Analysis of scRNA- and ScATAC-seq data}

Sample demultiplexing and barcode processing of scRNA-seq data was conducted with the Cell Ranger pipeline from 10x Genomics. For ESCs, quality filtering was conducted by selecting only cells within a certain percentage of mitochondrial reads $(2.5 \%<$ accepted cells $<7.5 \%)$ and number of detected genes $(2,000<$ accepted cells $<6,500)$, yielding 1,332 cells for time point $0 \mathrm{~h}, 2,085$ cells for $1 \mathrm{~h}$ and 4,825 for $6 \mathrm{~h}$ of IFN $\beta$ stimulation. For MEFs, quality filtering was conducted by selecting only cells within a certain percentage of mitochondrial reads $(0.5 \%<$ accepted cells $<7.5$ $\%)$ and number of detected genes $(1,250<$ accepted cells $<6,500)$, yielding 9,771 cells for time point $0 \mathrm{~h}, 10,186$ cells for $1 \mathrm{~h}$ and 7,579 for $6 \mathrm{~h}$ of IFN $\beta$ stimulation. Further analysis was done using the $\mathrm{R}$ package Seurat (Stuart et al, 2019). The scATAC-seq data were demultiplexed and aligned with Cell Ranger ATAC count (10x Genomics) using the provided mouse mm10 reference. Further processing of the data was conducted with ArchR (Granja et al, 2021). Cells were filtered using a minimal and maximal threshold for number of fragments $\left(10^{3.5}\right.$ and $10^{5}$, respectively), a TSS ratio above 4 and a ratio of fragments in blacklisted genomic regions to all fragments below 0.0225 (ESCs) and 0.016 (MEFs). Co-accessibility between genomic regions was separately calculated for cell types and treatment conditions adjusting the ArchR framework to single-cell resolution without aggregation of cells. The degree of co-accessibility in the background was determined by randomly shuffling the accessibility values over cells and peaks as described previously (Mallm et al, 2019). The $99^{\text {th }}$ percentile of the maximum shuffled background co-accessibility score was used as a threshold to determine true co-accessible links. Co-accessible links were further evaluated by percent of accessible cells in the linked peak pairs.

\section{Data access}

The data and computer code produced in this study are available from the following sources: All original sequencing and relevant processed data have been deposited under GSE160764 at Gene Expression Omnibus (https://www.ncbi.nlm.nih.gov/geo/). Software used for data analysis for the different sequencing readouts is listed in Supplementary Table S5.

\section{Author contributions}

Study design: KR, MM, FE

Acquisition of data: MM, CB, KMO, JPM, JH

Analysis of data: MM, IL, KMO, FE, LK, NK

Drafting of manuscript: MM, KR

Manuscript reviewing: all authors

Supervision and coordination: KR 


\section{Acknowledgments}

We thank Jorge Trojanowski, Sabrina Schumacher and Katharina Bauer for discussions and help and Mario Köster and Hansjörg Hauser for providing the IFN $\beta$ over-expression cell line. This work was supported by grants TRR179 (Z03) and Ri1283/14-1 of the German Research Foundation (DFG) to KR. We thank the DKFZ High Throughput Sequencing and the Omics IT and Data Management core facilities for support and services and the Central Animal Laboratory for preparation of mouse fibroblast cells. Additional data storage at SDS@hd was supported by the Ministry of Science, Research and the Arts Baden-Württemberg and the DFG through grants INST 35/1314-1 FUGG and INST 35/1503-1 FUGG.

\section{References}

Au-Yeung N, Horvath CM (2018) Transcriptional and chromatin regulation in interferon and innate antiviral gene expression. Cytokine Growth Factor Rev 44: 11-17

Au-Yeung N, Mandhana R, Horvath CM (2013) Transcriptional regulation by STAT1 and STAT2 in the interferon JAK-STAT pathway. JAKSTAT 2: e23931

Begitt A, Droescher M, Meyer T, Schmid CD, Baker M, Antunes F, Knobeloch KP, Owen MR, Naumann R, Decker T, Vinkemeier U (2014) STAT1-cooperative DNA binding distinguishes type 1 from type 2 interferon signaling. Nat Immunol 15: 168-176

Bernstein BE, Mikkelsen TS, Xie X, Kamal M, Huebert DJ, Cuff J, Fry B, Meissner A, Wernig M, Plath K, Jaenisch R, Wagschal A, Feil R, Schreiber SL, Lander ES (2006) A bivalent chromatin structure marks key developmental genes in embryonic stem cells. Cell 125: 315-326

Bibel M, Richter J, Lacroix E, Barde YA (2007) Generation of a defined and uniform population of CNS progenitors and neurons from mouse embryonic stem cells. Nat Protoc 2: 1034-1043

Blaszczyk K, Olejnik A, Nowicka H, Ozgyin L, Chen Y-L, Chmielewski S, Kostyrko K, Wesoly J, Balint BL, Lee C-K, Bluyssen HAR (2015) STAT2/IRF9 directs a prolonged ISGF3-like transcriptional response and antiviral activity in the absence of STAT1. Biochem J 466: 511-524

Borden EC (2019) Interferons alpha and beta in cancer: therapeutic opportunities from new insights. Nat Rev Drug Discov 18: 219-234

Chen K, Liu J, Cao X (2017) Regulation of type I interferon signaling in immunity and inflammation: A comprehensive review. J Autoimmun 83: 1-11

Cheng Y, He C, Wang M, Ma X, Mo F, Yang S, Han J, Wei X (2019) Targeting epigenetic regulators for cancer therapy: mechanisms and advances in clinical trials. Signal Transduct Target Ther 4: 62

Christova R, Jones T, Wu PJ, Bolzer A, Costa-Pereira AP, Watling D, Kerr IM, Sheer D (2007) PSTAT1 mediates higher-order chromatin remodelling of the human MHC in response to IFNgamma. J Cell Sci 120: 3262-3270

Creyghton MP, Cheng AW, Welstead GG, Kooistra T, Carey BW, Steine EJ, Hanna J, Lodato MA, Frampton GM, Sharp PA, Boyer LA, Young RA, Jaenisch R (2010) Histone H3K27ac separates active from poised enhancers and predicts developmental state. $P$ Natl Acad Sci USA 107: 2193121936

Cusack M, King HW, Spingardi P, Kessler BM, Klose RJ, Kriaucionis S (2020) Distinct contributions of DNA methylation and histone acetylation to the genomic occupancy of transcription factors. Genome Res 30: 1393-1406

D'Angelo W, Acharya D, Wang R, Wang J, Gurung C, Chen B, Bai F, Guo YL (2016) Development of Antiviral Innate Immunity During In Vitro Differentiation of Mouse Embryonic Stem Cells. Stem Cells Dev 25: 648-659 
de Veer MJ, Holko M, Frevel M, Walker E, Der S, Paranjape JM, Silverman RH, Williams BR (2001) Functional classification of interferon-stimulated genes identified using microarrays. $J$ Leukoc Biol 69: 912-920

Der SD, Zhou A, Williams BR, Silverman RH (1998) Identification of genes differentially regulated by interferon alpha, beta, or gamma using oligonucleotide arrays. Proc Natl Acad Sci USA 95: 15623-15628

Dobin A, Davis CA, Schlesinger F, Drenkow J, Zaleski C, Jha S, Batut P, Chaisson M, Gingeras TR (2013) STAR: ultrafast universal RNA-seq aligner. Bioinformatics 29: 15-21

Encode Project Consortium (2012) An integrated encyclopedia of DNA elements in the human genome. Nature 489: 57-74

Erdel F, Rademacher A, Vlijm R, Tunnermann J, Frank L, Weinmann R, Schweigert E, Yserentant K, Hummert J, Bauer C, Schumacher S, Al Alwash A, Normand C, Herten DP, Engelhardt J, Rippe K (2020) Mouse Heterochromatin Adopts Digital Compaction States without Showing Hallmarks of HP1-Driven Liquid-Liquid Phase Separation. Mol Cell 78: 236-249 e237

Ernst J, Kheradpour P, Mikkelsen TS, Shoresh N, Ward LD, Epstein CB, Zhang X, Wang L, Issner R, Coyne M, Ku M, Durham T, Kellis M, Bernstein BE (2011) Mapping and analysis of chromatin state dynamics in nine human cell types. Nature 473: 43-49

Feldmann A, Dimitrova E, Kenney A, Lastuvkova A, Klose RJ (2020) CDK-Mediator and FBXL19 prime developmental genes for activation by promoting atypical regulatory interactions. Nucleic Acids Res 48: 2942-2955

Frank L, Weinmann R, Erdel F, Trojanowski J, Rippe K (2021) Transcriptional Activation of Heterochromatin by Recruitment of dCas9 Activators. Methods Mol Biol 2351: 307-320

Gonzalez-Navajas JM, Lee J, David M, Raz E (2012) Immunomodulatory functions of type I interferons. Nat Rev Immunol 12: 125-135

Gorisch SM, Wachsmuth M, Toth KF, Lichter P, Rippe K (2005) Histone acetylation increases chromatin accessibility. J Cell Sci 118: 5825-5834

Granja JM, Corces MR, Pierce SE, Bagdatli ST, Choudhry H, Chang HY, Greenleaf WJ (2021) ArchR is a scalable software package for integrative single-cell chromatin accessibility analysis. Nature Genetics 53: 403-411

Guo YL (2017) Utilization of different anti-viral mechanisms by mammalian embryonic stem cells and differentiated cells. Immunol Cell Biol 95: 17-23

Guo YL, Carmichael GG, Wang R, Hong X, Acharya D, Huang F, Bai F (2015) Attenuated Innate Immunity in Embryonic Stem Cells and Its Implications in Developmental Biology and Regenerative Medicine. Stem Cells 33: 3165-3173

Heinz S, Benner C, Spann N, Bertolino E, Lin YC, Laslo P, Cheng JX, Murre C, Singh H, Glass CK (2010) Simple combinations of lineage-determining transcription factors prime cis-regulatory elements required for macrophage and B cell identities. Mol Cell 38: 576-589

Hoffmann HH, Schneider WM, Rice CM (2015) Interferons and viruses: an evolutionary arms race of molecular interactions. Trends Immunol 36: 124-138

Hu X, Li J, Fu M, Zhao X, Wang W (2021) The JAK/STAT signaling pathway: from bench to clinic. Signal Transduct Target Ther 6: 402

Huang M, Qian F, Hu Y, Ang C, Li Z, Wen Z (2002) Chromatin-remodelling factor BRG1 selectively activates a subset of interferon-alpha-inducible genes. Nat Cell Biol 4: 774-781

Ivashkiv LB, Donlin LT (2014) Regulation of type I interferon responses. Nat Rev Immunol 14: 3649 
Karr JP, Ferrie JJ, Tjian R, Darzacq X (2022) The transcription factor activity gradient (TAG) model: contemplating a contact-independent mechanism for enhancer-promoter communication. Genes Dev 36: 7-16

Langmead B, Salzberg SL (2012) Fast gapped-read alignment with Bowtie 2. Nat Methods 9: 357359

Lazear HM, Schoggins JW, Diamond MS (2019) Shared and Distinct Functions of Type I and Type III Interferons. Immunity 50: 907-923

Lee TI, Young RA (2013) Transcriptional regulation and its misregulation in disease. Cell 152: 12371251

Lehnertz B, Ueda Y, Derijck AA, Braunschweig U, Perez-Burgos L, Kubicek S, Chen T, Li E, Jenuwein T, Peters AH (2003) Suv39h-mediated histone H3 lysine 9 methylation directs DNA methylation to major satellite repeats at pericentric heterochromatin. Curr Biol 13: 1192-1200

Li B, Dewey CN (2011) RSEM: accurate transcript quantification from RNA-Seq data with or without a reference genome. BMC Bioinformatics 12: 323

Li K, Liu Y, Cao H, Zhang Y, Gu Z, Liu X, Yu A, Kaphle P, Dickerson KE, Ni M, Xu J (2020) Interrogation of enhancer function by enhancer-targeting CRISPR epigenetic editing. Nat Commun 11: 485

Liu H, Kang H, Liu R, Chen X, Zhao K (2002) Maximal induction of a subset of interferon target genes requires the chromatin-remodeling activity of the BAF complex. Mol Cell Biol 22: 6471-6479

Local A, Huang H, Albuquerque CP, Singh N, Lee AY, Wang W, Wang C, Hsia JE, Shiau AK, Ge K, Corbett KD, Wang D, Zhou H, Ren B (2018) Identification of H3K4me1-associated proteins at mammalian enhancers. Nat Genet 50: 73-82

Love MI, Huber W, Anders S (2014) Moderated estimation of fold change and dispersion for RNAseq data with DESeq2. Genome Biol 15: 550

Lu Y, Stuart JH, Talbot-Cooper C, Agrawal-Singh S, Huntly B, Smid AI, Snowden JS, Dupont L, Smith GL (2019) Histone deacetylase 4 promotes type I interferon signaling, restricts DNA viruses, and is degraded via vaccinia virus protein C6. P Natl Acad Sci USA 116: 11997-12006

Maillard PV, Ciaudo C, Marchais A, Li Y, Jay F, Ding SW, Voinnet O (2013) Antiviral RNA interference in mammalian cells. Science 342: $235-238$

Mallm JP, Iskar M, Ishaque N, Klett LC, Kugler SJ, Muino JM, Teif VB, Poos AM, Grossmann S, Erdel F, Tavernari D, Koser SD, Schumacher S, Brors B, Konig R, Remondini D, Vingron M, Stilgenbauer S, Lichter P, Zapatka M, Mertens D, Rippe K (2019) Linking aberrant chromatin features in chronic lymphocytic leukemia to transcription factor networks. Mol Syst Biol 15: e8339

Mallm JP, Windisch P, Biran A, Gal Z, Schumacher S, Glass R, Herold-Mende C, Meshorer E, Barbus M, Rippe K (2020) Glioblastoma initiating cells are sensitive to histone demethylase inhibition due to epigenetic deregulation. Int J Cancer 146: 1281-1292

Mohammad HP, Barbash O, Creasy CL (2019) Targeting epigenetic modifications in cancer therapy: erasing the roadmap to cancer. Nat Med 25: 403-418

Molitor J, Mallm JP, Rippe K, Erdel F (2017) Retrieving Chromatin Patterns from Deep Sequencing Data Using Correlation Functions. Biophys J 112: 473-490

Morey L, Helin K (2010) Polycomb group protein-mediated repression of transcription. Trends Biochem Sci 35: 323-332

Mostafavi S, Yoshida H, Moodley D, LeBoite H, Rothamel K, Raj T, Ye CJ, Chevrier N, Zhang SY, Feng T, Lee M, Casanova JL, Clark JD, Hegen M, Telliez JB, Hacohen N, De Jager PL, Regev A, Mathis D, Benoist C, Immunological Genome Project C (2016) Parsing the Interferon Transcriptional Network and Its Disease Associations. Cell 164: 564-578 
Nusinzon I, Horvath CM (2003) Interferon-stimulated transcription and innate antiviral immunity require deacetylase activity and histone deacetylase 1. P Natl Acad Sci USA 100: 14742-14747

Ostuni R, Piccolo V, Barozzi I, Polletti S, Termanini A, Bonifacio S, Curina A, Prosperini E, Ghisletti S, Natoli G (2013) Latent enhancers activated by stimulation in differentiated cells. Cell 152: 157171

Platanitis E, Demiroz D, Schneller A, Fischer K, Capelle C, Hartl M, Gossenreiter T, Muller M, Novatchkova M, Decker T (2019) A molecular switch from STAT2-IRF9 to ISGF3 underlies interferon-induced gene transcription. Nat Commun 10: 2921

Platanitis E, Grüner S, Geetha ARSJ, Boccuni L, Vogt A, Novachkova M, Sommer A, Müller M, Decker T (2021) Interferons reshape the 3D conformation and accessibility of macrophage chromatin. bioRxiv: 2021.2003.2009.434568

Poirier EZ, Buck MD, Chakravarty P, Carvalho J, Frederico B, Cardoso A, Healy L, Ulferts R, Beale $R$, Reis e Sousa C (2021) An isoform of Dicer protects mammalian stem cells against multiple RNA viruses. Science 373: 231-236

Robertson G, Hirst M, Bainbridge M, Bilenky M, Zhao Y, Zeng T, Euskirchen G, Bernier B, Varhol R, Delaney A, Thiessen N, Griffith OL, He A, Marra M, Snyder M, Jones S (2007) Genome-wide profiles of STAT1 DNA association using chromatin immunoprecipitation and massively parallel sequencing. Nat Methods 4: 651-657

Ross-Innes CS, Stark R, Teschendorff AE, Holmes KA, Ali HR, Dunning MJ, Brown GD, Gojis O, Ellis IO, Green AR, Ali S, Chin SF, Palmieri C, Caldas C, Carroll JS (2012) Differential oestrogen receptor binding is associated with clinical outcome in breast cancer. Nature 481: 389-393

Sa Ribero M, Jouvenet N, Dreux M, Nisole S (2020) Interplay between SARS-CoV-2 and the type I interferon response. PLoS Pathog 16: e1008737

Schwerk J, Koster M, Hauser H, Rohde M, Fulde M, Hornef MW, May T (2013) Generation of mouse small intestinal epithelial cell lines that allow the analysis of specific innate immune functions. PLoS One 8: e72700

Shogren-Knaak M, Ishii H, Sun JM, Pazin MJ, Davie JR, Peterson CL (2006) Histone H4-K16 acetylation controls chromatin structure and protein interactions. Science 311: 844-847

Singh H, Khan AA, Dinner AR (2014) Gene regulatory networks in the immune system. Trends Immunol 35: 211-218

Stanifer ML, Guo C, Doldan P, Boulant S (2020) Importance of Type I and III Interferons at Respiratory and Intestinal Barrier Surfaces. Front Immunol 11: 608645

Stanifer ML, Pervolaraki K, Boulant S (2019) Differential Regulation of Type I and Type III Interferon Signaling. Int J Mol Sci 20: 1445

Stark GR, Darnell JE, Jr. (2012) The JAK-STAT pathway at twenty. Immunity 36: 503-514

Stuart T, Butler A, Hoffman P, Hafemeister C, Papalexi E, Mauck WM, 3rd, Hao Y, Stoeckius M, Smibert P, Satija R (2019) Comprehensive Integration of Single-Cell Data. Cell 177: 1888-1902 e1821

Tang X, Gao JS, Guan YJ, McLane KE, Yuan ZL, Ramratnam B, Chin YE (2007) Acetylationdependent signal transduction for type I interferon receptor. Cell 131: 93-105

Teif VB, Vainshtein Y, Caudron-Herger M, Mallm JP, Marth C, Hofer T, Rippe K (2012) Genomewide nucleosome positioning during embryonic stem cell development. Nat Struct Mol Biol 19: 11851192

Testoni B, Vollenkle C, Guerrieri F, Gerbal-Chaloin S, Blandino G, Levrero M (2011) Chromatin dynamics of gene activation and repression in response to interferon alpha (IFN(alpha)) reveal new roles for phosphorylated and unphosphorylated forms of the transcription factor STAT2. J Biol Chem 286: 20217-20227 
Vahedi G, Takahashi H, Nakayamada S, Sun HW, Sartorelli V, Kanno Y, O'Shea JJ (2012) STATs shape the active enhancer landscape of T cell populations. Cell 151: 981-993

Villarino AV, Kanno Y, O'Shea JJ (2017) Mechanisms and consequences of Jak-STAT signaling in the immune system. Nat Immunol 18: 374-384

Wang R, Wang J, Acharya D, Paul AM, Bai F, Huang F, Guo YL (2014) Antiviral responses in mouse embryonic stem cells: differential development of cellular mechanisms in type I interferon production and response. J Biol Chem 289: 25186-25198

Wang R, Wang J, Paul AM, Acharya D, Bai F, Huang F, Guo YL (2013) Mouse embryonic stem cells are deficient in type I interferon expression in response to viral infections and double-stranded RNA. J Biol Chem 288: 15926-15936

Wang X, Hayes JJ (2008) Acetylation mimics within individual core histone tail domains indicate distinct roles in regulating the stability of higher-order chromatin structure. Mol Cell Biol 28: 227-236

Whyatt LM, Duwel A, Smith AG, Rathjen PD (1993) The responsiveness of embryonic stem cells to alpha and beta interferons provides the basis of an inducible expression system for analysis of developmental control genes. Mol Cell Biol 13: 7971-7976

Wu X, Dao Thi VL, Huang Y, Billerbeck E, Saha D, Hoffmann HH, Wang Y, Silva LAV, Sarbanes S, Sun T, Andrus L, Yu Y, Quirk C, Li M, MacDonald MR, Schneider WM, An X, Rosenberg BR, Rice CM (2018) Intrinsic Immunity Shapes Viral Resistance of Stem Cells. Cell 172: 423-438 e425

Xu S, Grullon S, Ge K, Peng W (2014) Spatial clustering for identification of ChIP-enriched regions (SICER) to map regions of histone methylation patterns in embryonic stem cells. Methods Mol Biol 1150: $97-111$

Yamawaki TM, Lu DR, Ellwanger DC, Bhatt D, Manzanillo P, Arias V, Zhou H, Yoon OK, Homann O, Wang S, Li CM (2021) Systematic comparison of high-throughput single-cell RNA-seq methods for immune cell profiling. BMC Genomics 22: 66

Zhang Y, Liu T, Meyer CA, Eeckhoute J, Johnson DS, Bernstein BE, Nusbaum C, Myers RM, Brown M, Li W, Liu XS (2008) Model-based analysis of ChIP-Seq (MACS). Genome Bio/ 9: R137 